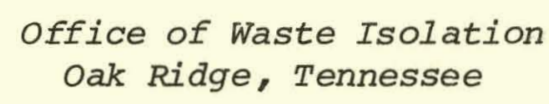

operated by

Union Carbide Corporation

Nuclear Division

for the

Energy Research and Development Administration

By

\author{
Gary D. Callahan \\ and \\ Arlo F. Fossum
}

February 1, 1977

This report was prepared by RE/SPEC Inc, , under Subcontract No. 89Y-22303C with Union Carbide Corporation, Nuclear Division, under Contract No. W-7405-eng-26 with the Energy Research and Development Administration. The subcontract was administered by the office of Waste Isolaliun, Union Carbide corporation, Nuclear Division.

\title{
RE/SPEC INC.
}

P. O. BOX 725 - RAPID CITY, S. O. 57101 - 605/343-7868 


\section{DISCLAIMER}

This report was prepared as an account of work sponsored by an agency of the United States Government. Neither the United States Government nor any agency Thereof, nor any of their employees, makes any warranty, express or implied, or assumes any legal liability or responsibility for the accuracy, completeness, or usefulness of any information, apparatus, product, or process disclosed, or represents that its use would not infringe privately owned rights. Reference herein to any specific commercial product, process, or service by trade name, trademark, manufacturer, or otherwise does not necessarily constitute or imply its endorsement, recommendation, or favoring by the United States Government or any agency thereof. The views and opinions of authors expressed herein do not necessarily state or reflect those of the United States Government or any agency thereof. 


\section{DISCLAIMER}

Portions of this document may be illegible in electronic image products. Images are produced from the best available original document. 
DATA INPUT MANUAL FOR RSI/TRANCO:

\title{
A FINITE ELEMENT HEAT CONDUCTION COMPUTER PROGRAM
}

\author{
Submitted To \\ Office of Waste Isolation \\ oak Ridge, Tennessee \\ operated by \\ Union Carbide Corporation \\ Nuclear Division \\ for the
}

Energy Research and Development Administration

$B y$
Gary D. Callahan
and
Arlo F. Fossum

of

$R E / S P E C$ InC.

P. O. DOX 725

Rapid City, South Dakota

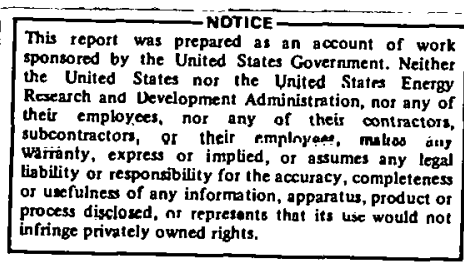

This report was prepared as an account of work the United States nor the United Stant. Neither Research and Uevelopment Administration, not any of subcontractora, or their of infringe privately owned rights.

February 1, 1977

This report was prepared as an account of work sponsored by the United States Government. Neither the United States nor the Energy Research and Development Administration, nor any of their employees, nor any of their contractors, subcontractors, or their employees, makes any warranty, express or implied, or assumes any legal liability or responsibility for the accuracy, completeness or nsefulness of any information, apparatus, product or process disclosed, or represents that its use would not infringe privately owned rights. Any mention of trade names in this report is for reference purposes only and should not be construed as any manner of endorsement. 


\section{FOREWORD}

This report was prepared under a subcontract with Union Carbide corporation, Nuclear Division, an ERDA contractor. The subcontract was administered by the office of Waste Isolation and is part of the National Waste Terminal Storage (NWTS) Program. The principal objective of the NWTS Program is to provide facilities in various deep geologic formations at multiple locations in the United States which will safely dispose of commercial radioactive waste, which must be delivered to a Federal Repository for terminal storage. Some of the expected wastes produce both heat and radioactivity. This situation leads to many unique problems in rock mechanics. This report addresses a particular problem relative to the Rock Mechanics Program.

The overall objective of the OWI Rock Mechanics Program is to predict the response of a rock mass hosting a waste repository during its constructizn and operation, as well as the post-operational phase. The operational phase is expected to be approximately 20 years while the post-operational phase will last until the repository no longer poses any potential hazard to mankind, a period that may last several hundred thousand years. The Rock Mechanics Program is concerned with near field effects on mine stability as well as far field effects relative to the overall integrity of the geologic containment of waste.

In order to accomplish the objectives of the Rock Mechanics Program, numerical simulation, laboratory (including bench scale;, and field studies are under way. The laboratory and field studies provide input to the numerical simulations and also the opportunity for validation of the predictive capabilities of the computer codes. Ultimately, the computer codes will provide the predicted response of the host rock mass and thereby form an essential part of the overall Rock Mechanics Program.

This report provides information on input data as well as FORTRAN 1isting of RSI/TRANCO: A Finite Element Heat Conduction Computer Program.

Documentation of all computer codes used in the NWTS Program is important in order to provide for continuity in the program and acceptance by both. the scientific community and the public. 
1. INTRODUCTION

2. INPUT DATA DESCRIPTION

3. EXAMPLE PROBLEM 15

3.1. Problem Description 15

3.2. Finite Element Discretization 17

3.3. Input Data 18

3.4. Results 21

$\begin{array}{ll}R E F E R E N C E S & 28\end{array}$

APPENDIX A: RSI/TRANCO Fortran Listing: A-1

\section{LIST OF FIGURES}

Figure No.

$\underline{\text { Page }}$

1 Segment of a typical finite element mesh utilizing eight noded isoparametric elements:

\section{ACKNOWLEDGEMENTS}

The technical contents of this report have been reviewed by Dr. Paul F. Gnirk and Mr. Joe L. Ratigan. The report was typed by Ms. Julie S. Annicchiarico and Ms. Elizabeth A. Speer. 


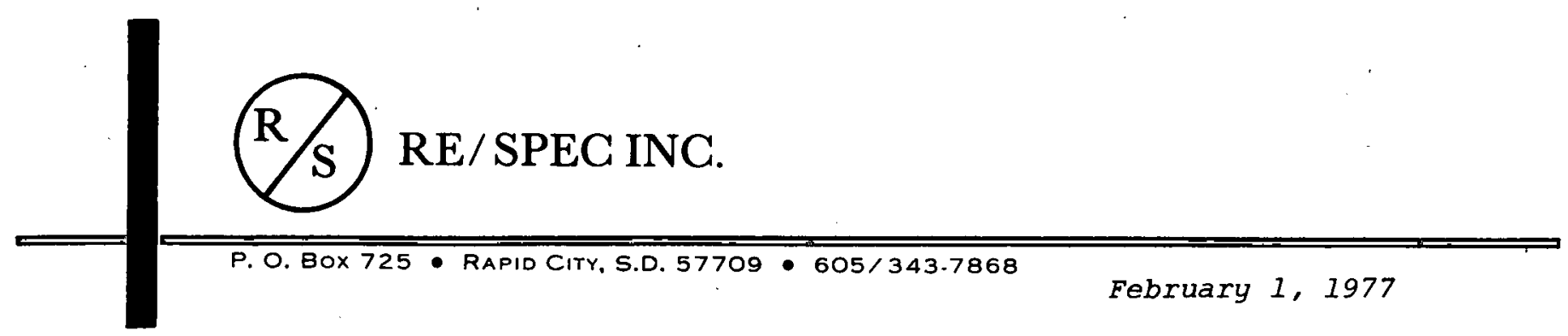

MEMORANDUM REPORT RSI-0049

TO:

Office of Waste Isolation

Building 9102-2

P. O. BOX $Y$

Oak Ridge, TN 37830

FROM: $\quad$ Mr. Gary D. Callahan

Dr. Arlo F. Fossum

RE/SPEC InC.

P. O. Box 725

Rapid City, SD 57701

SUBJECT: Data Input Manual for RSI/TRANCO: A Finite Element Heat

Transfer Computer Program (Ref.: Union Carbide Corporation, Nuclear Division Subcontract No. 89Y-22303 (RSI-001000/FY77)).

1. INTRODUCTION

Program: $\quad$ RSI/TRANCO

Function: RSI/TRANCO solves plane isotropic or anisotropic transient or steady state heat transfer problems. Boundary condition types include fixed temperature, constant flux, and convective. In addition, constant internal heat sources and constant or temperature dependent thermal properties are included.

Output: Input data, if desired, and the nodal values of the temperature field are printed.

Arbitrarily shaped, plane, boundary value problems in heat transfer may be solved with RSI/TRANCO utilizing eight noded isoparametric elements and six noded subparametric (i.e. geometry polynomial is of a lower order than the displacement polynomial) triangular elements or a combination of both. For a discussion of the finite element method, the reader is directed to References 1 and 2 .

Figure 1 presents a segment of a finite element mesh which is representative of most problems and defines certain items which may not be commn terminology. 


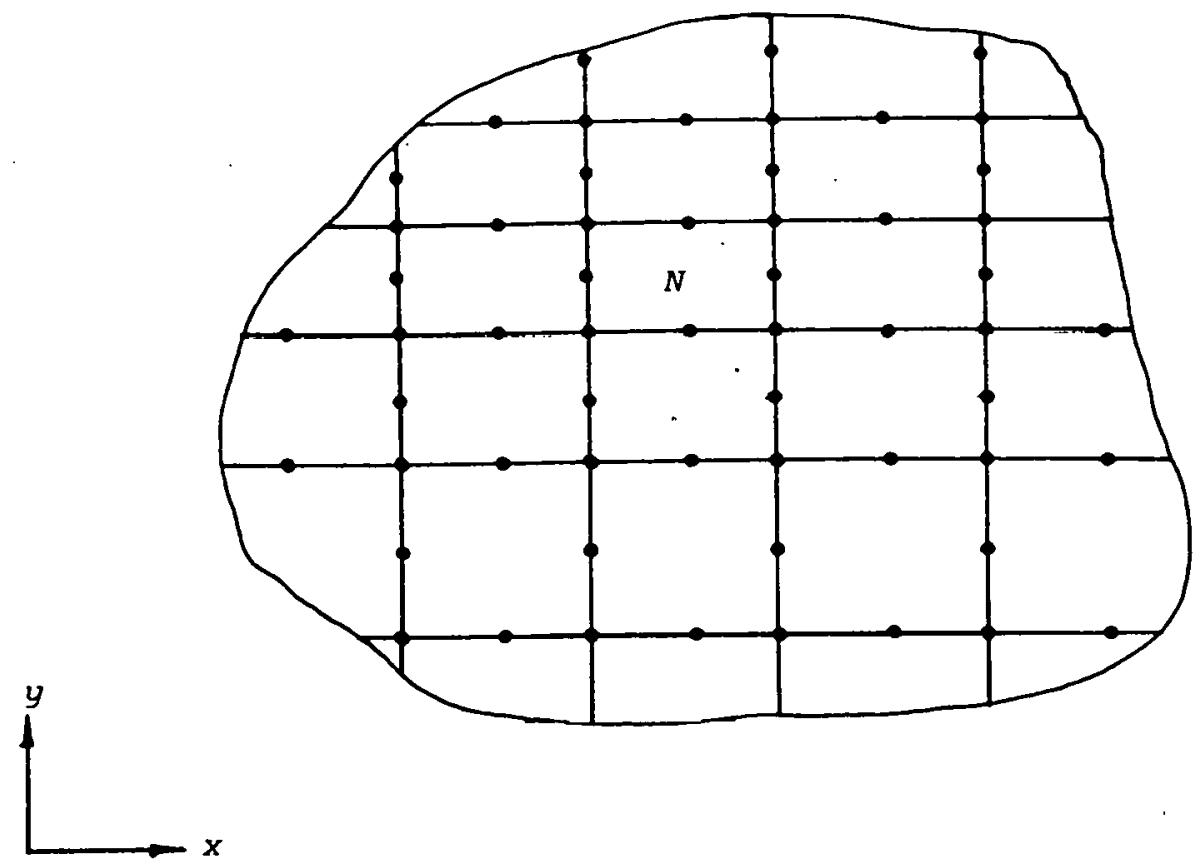

(a) Segment from a Typical Finite Element Discretization.

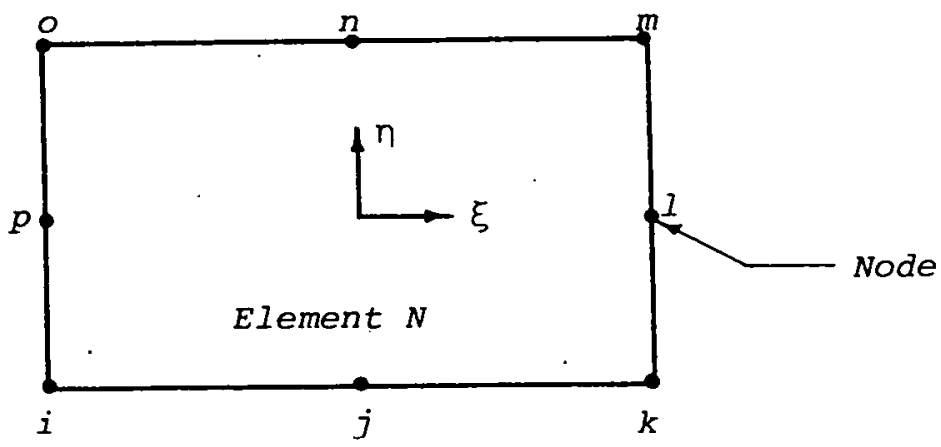

(b) Typical Eight-Noded Isoparametric Element.

$\begin{array}{ll}\text { Primary Nodes } & -i, k, m, 0 \\ \text { Secondary Nodes } & -j, I, n, p \\ \text { Global Coordinate Axes } & -x, y \\ \text { Local Coordinate Axes } & -n, \xi\end{array}$

Figure 1. Seyment of a typical finfte element mesh utilizing eight noded isoparametric elements. 
In Section 2, each required data card type is described. The bulk of the input data is associated with card types 8 and 9 which are required to describe the finite element mesh. At times, one may find advantages in writing a small computer program to generate the nodal coordinates and element nodal identifications automatically.

In the final section, an example problem is presented to illustrate the mesh preparation, data input, and sample output. A fortran listing of the computer program is given in the Appendix, along with current program limits. 


\section{INPUT DATA DESCRIPTION}

The following pages ilustrate and describe the required data for running various heat transfer problems utilizing RSI/TRANCO. Each data card type is discussed separately and requires no further discussion at this point.

The units on various parameters may be any set of constant units. For example:

$$
\begin{aligned}
& \text { nodal coordinates - ft. } \\
& \text { thermal conductivity } \\
& -\frac{B t u}{f t-h r-o_{F}} \\
& \text { specific heat } \\
& -\frac{B t u}{I b_{m}-O_{F}} \\
& \text { density } \\
& -\frac{1 b_{m}}{f t^{3}} \\
& \text { convective film coefficient }-\frac{B t u}{f t^{2}-h r-{ }^{\circ} F} \\
& \text { time-step - } \quad \text { hr. } \\
& \text { heat generation rate } \\
& -\frac{B t u}{h r-f t^{3}}
\end{aligned}
$$

Using the above set of units, the nodal temperatures are output in degrees Fahrenheit.

In the following text, dimcnoions arc given in terms of mass (M), length $(L)$, time $(t)$, and temperature $(T)$.

DATA CARD I

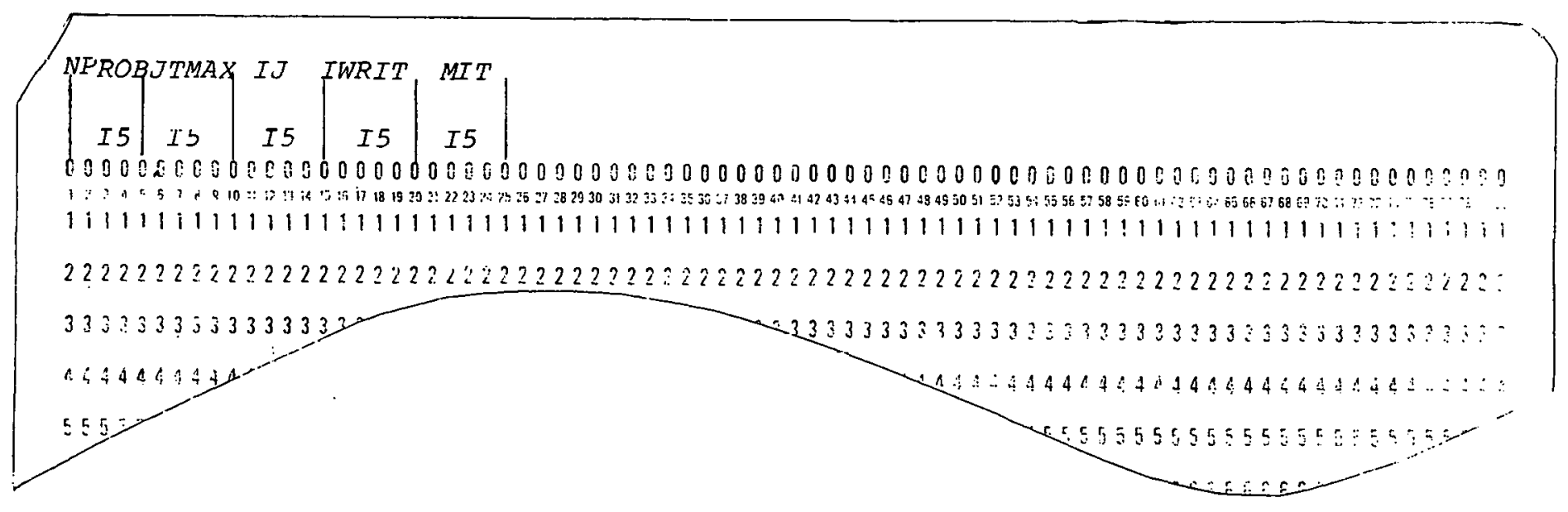


where:

$N P R O B$ is greater than one for multiple runs.

JTMAX represents the number of time steps.

IJ

Constitutes a convective boundary control parameter

$$
\begin{array}{ll}
I J=0 & \text { for no convective boundaries } \\
I J=1 & \text { for convective boundaries }
\end{array}
$$

IWRIT Controls output.

$$
\begin{array}{ll}
\text { IWRIT }=1 & \text { prints nodal temperatures } \\
I W R I T=2 & \begin{array}{l}
\text { prints nodal temperatures and punches } \\
\text { the temperature field for the final } \\
\text { time step. }
\end{array}
\end{array}
$$

MIT A parameter offering restart capability $(M I T<0)$. Set $M I T=0$ for other runs.

For example, if one desires to change the time step, material properties, or temperature dependent conductivities, enter NPROB $>1$. When JTMAX time steps have been executed, control is transferred to the beginning of the program and data card types 1-7 will be read and therefore must be included following the regular data set with the desired changes. In the second data set, enter $M I T<0$ indicating a continuation of the first problem.

When the restart parameter is utilized, the existing temperature field becomes the initial temperature field for the continuing problem.

Note that to use the MIT option NPROB (Data Card 1) must be greater than one in the first data set and in subsequent sets until further restarts are not anticipated.

DATA CARD 2

\begin{tabular}{l|l|l|}
$D E L T$ & $I P R$ & $I T D E P$ \\
$F 15.6$ & $I 5$ & $I 5$
\end{tabular}

00000000000000000000000000000000000000000000000000000000000000000000000000003000

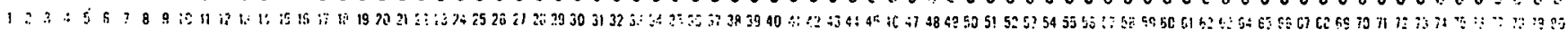

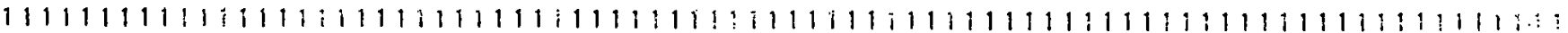

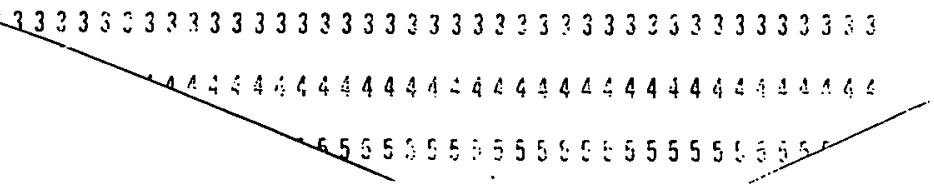


where:

$$
\begin{aligned}
& \text { DELT is the magnitude of the time step, }(t) \text {. } \\
& \text { IPR is the print interval on time steps (Default }=1 \text { ). } \\
& \text { For example, IPR }=3 \text { results in every third time } \\
& \text { step being printed. } \\
& \text { ITDEP controls thermal properties } \\
& \text { ITDEP }=0 \text { constant thermal properties } \\
& \text { ITDEP }=1 \text { temperature dependent thermal properties }
\end{aligned}
$$

\section{DATA CARD 3}

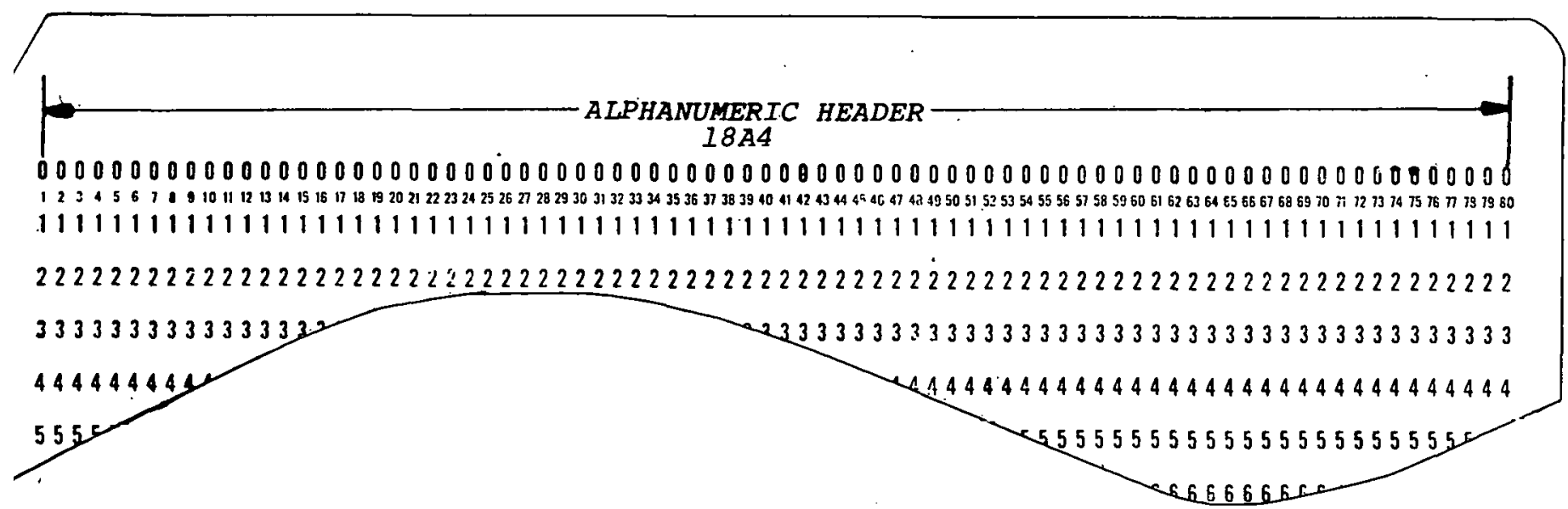

Header card identifies the computer run. Only one card is permitted.

DATA CARD 4

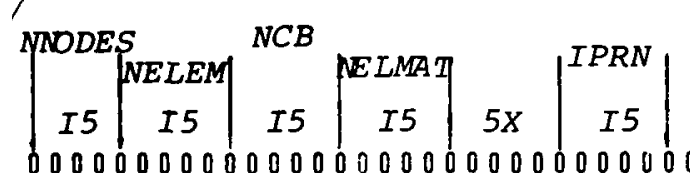

00000000000000000000000000000000000000000000000000000000000000000000000000000000

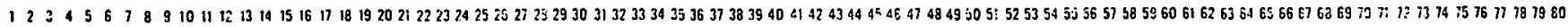
1111111111111111111111111111111111111111111111111111111111111111111111111111

22222222222222222222222222222222222222222222222222222222222222222222222222222222

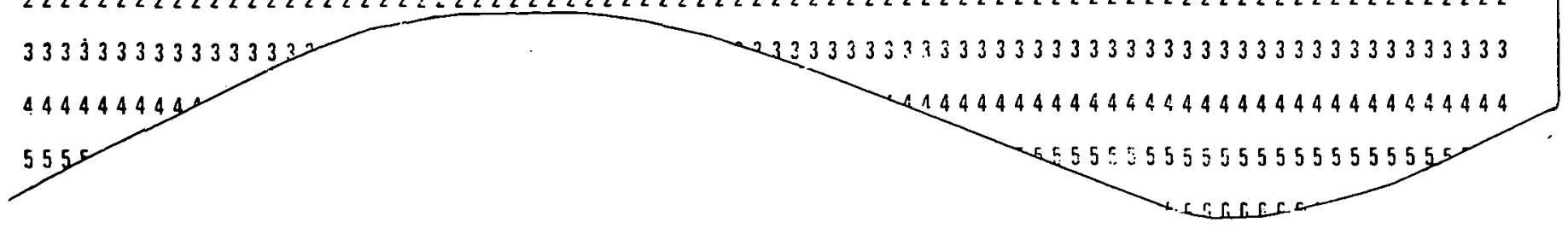


where:

$$
\begin{aligned}
& \text { NNODES is the number of nodal points. } \\
& \text { NELEM is the number of elements. } \\
& N C B \text { equals the number of convective boundaries ( } 3 \text { nodes } \\
& \text { per boundary). See Card Type I (IJ), and Card Type } 10 . \\
& \text { NELMAT is the number of different material types. } \\
& \text { IPRN equals } I \text { to print input data. IPRN }=0 \text { results } \\
& \text { in partial elimination of data printout. }
\end{aligned}
$$

$D A T A C A R D(S) \quad 5$

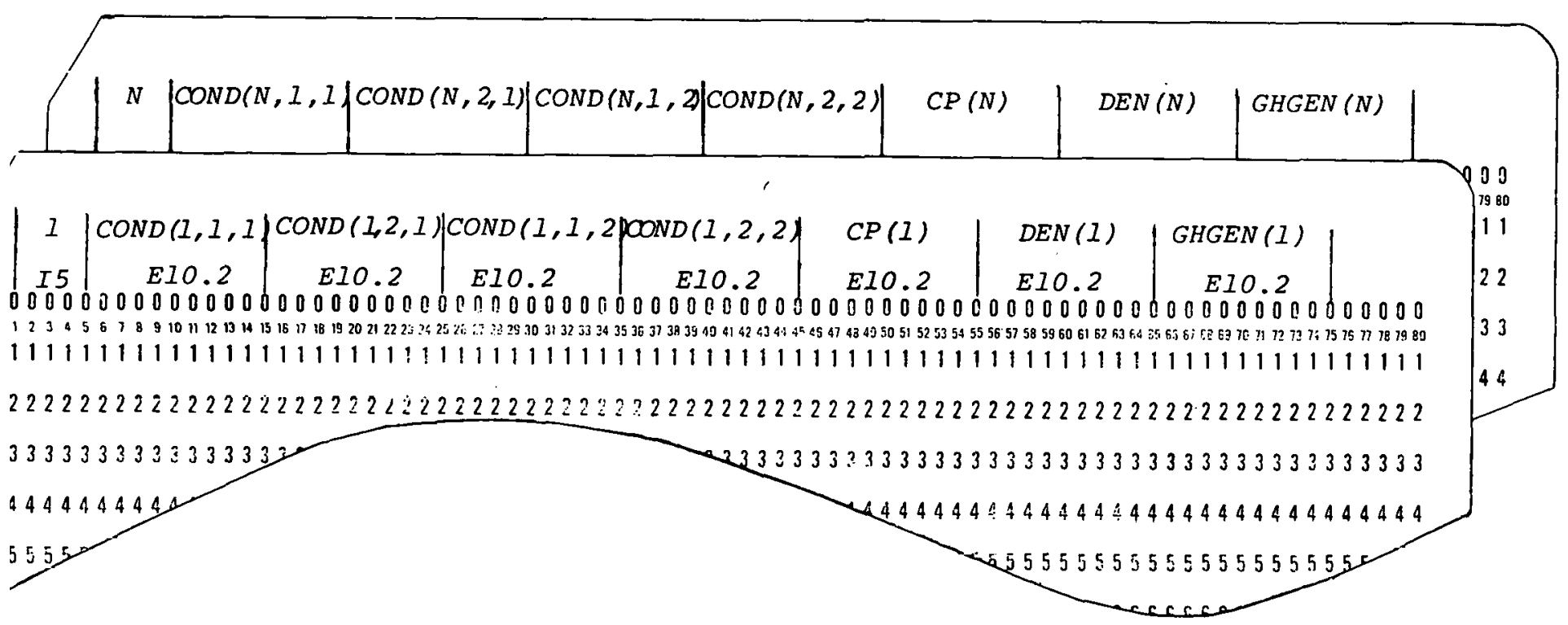

where:

$N \quad$ is an integer identifying the material type

(up to NELMAT different types)

$\operatorname{COND}(N, I, J)$ is the thermal conductivity of material type $N\left(M L / t^{3} T\right)$

$$
\begin{aligned}
& \operatorname{COND}(N, 1,1)=k_{x x}^{N} \\
& \operatorname{COND}(N, 2,1)=k_{y x}^{N} \\
& \operatorname{COND}(N, 1,2)=k_{x y}^{N} \\
& \operatorname{COND}(N, 2,2)=k_{y \varphi}^{N}
\end{aligned}
$$


$C P(N) \quad$ is the specific heat of material type $N\left(L^{2} / t^{2} T\right)$

$D E N(N) \quad$ is the density $\left(M / L^{3}\right)$ of material type $N$

GHGEN (N) is the heat generation rate $\left(M / L t^{3}\right)$ of material type $N$

Data card types 6 and 7 pertain to the temperature dependent conductivities. For ITDEP (data card 2) not equal to one, omit these cards; consequently constant properties are assumed.

Currently the computer program computes up to second order temperature dependence which may vary for each element. For example, for any one particular element $N$ of matertal type $I$.

$$
X \operatorname{COND}(J, K)=\operatorname{SLOP} 2(I, J, K) * T^{2}+\operatorname{SLOPE}(I, J, K) * T+\operatorname{COND}(I, J, K)(J, K=1,2)
$$

where:

$$
\begin{aligned}
& \text { XCOND }=\text { the modified conductivity for element } N \text { at temperature } T \\
& \text { SLOP2 = the change of the conductivity with the square of the } \\
& \text { temperature (acceleration of conductivity with respect } \\
& \text { to temperature of the characteristic temperature } \\
& \text { dependent conductivity curve) for material type } I \text {. } \\
& \text { sLop } 2 \text { parameters are defined in data card(s) } 7 . \\
& \text { SLOPE }=\text { the change of the conductivity with temperature. For } \\
& \text { example, if a linear dependence of conductivity with } \\
& \text { respect to temperature is desired, SLOP2 parameters } \\
& \text { would be zero and SLOPE would represent the slope of } \\
& \text { the characteristic conductivity line. SLOPE parameters } \\
& \text { are defined in data card(s) } 6 .
\end{aligned}
$$

$\underline{D A T A} \operatorname{CARD}(S) \quad 6$

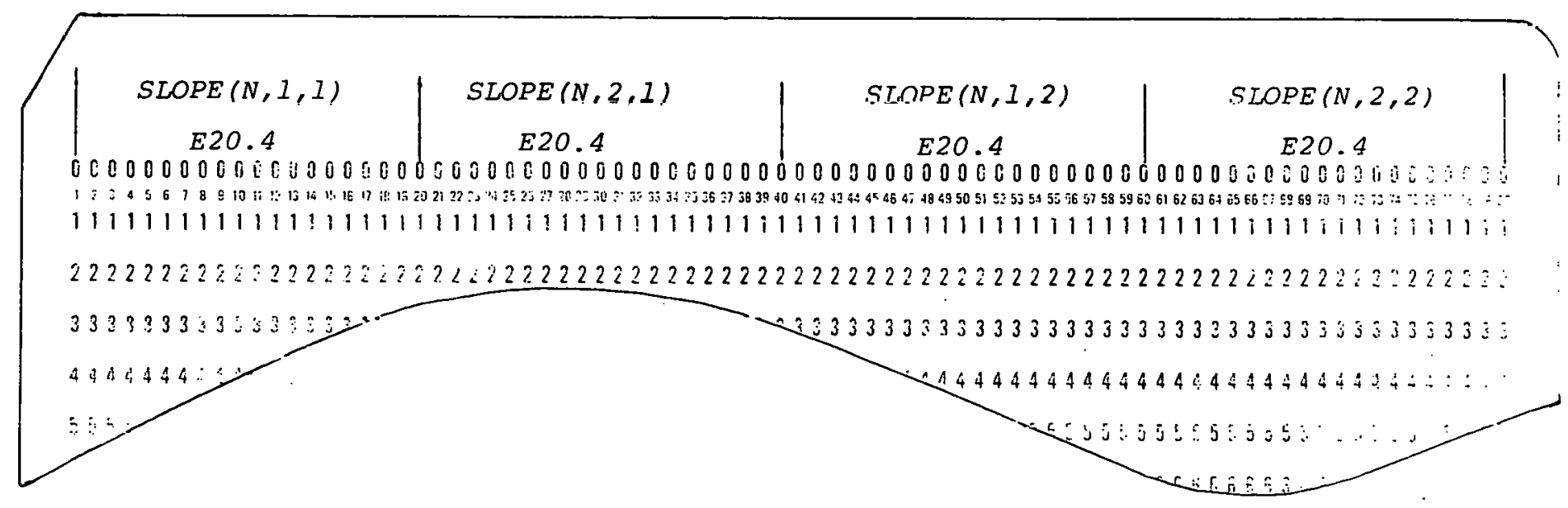


One SLOPE card is supplied for each material type (NELMAT, data $\operatorname{card} 4)$.

DATA CARD(S) ?

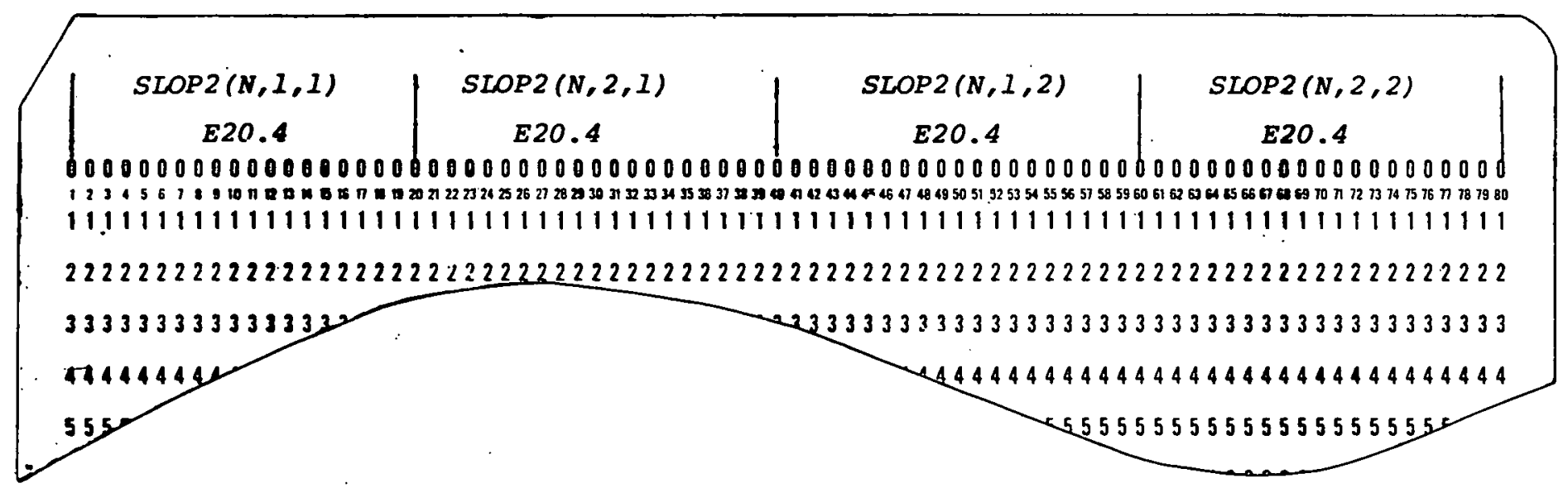

One SLOP2 card is supplied for each material type (NELMAT, data card 4).

DATA CARDS 8 consist of the global nodal coordinates of the finite element mesh. When nodal point cards are omitted, the coordinates are generated at equal intervals between the nodal point data supplied, say nodes $n l$ and $n n$. In addition, with JIG $=0$ on the ending nodal point card of the generation sequence (nn in this case) ITYPN and TCONS are set equal to zero for each generated nodal point. However, with JIG > O, ITYPN and TCONS are set equal to the values provided for the first node in the generation sequence (nI in this case). DATA CARD(S) 8

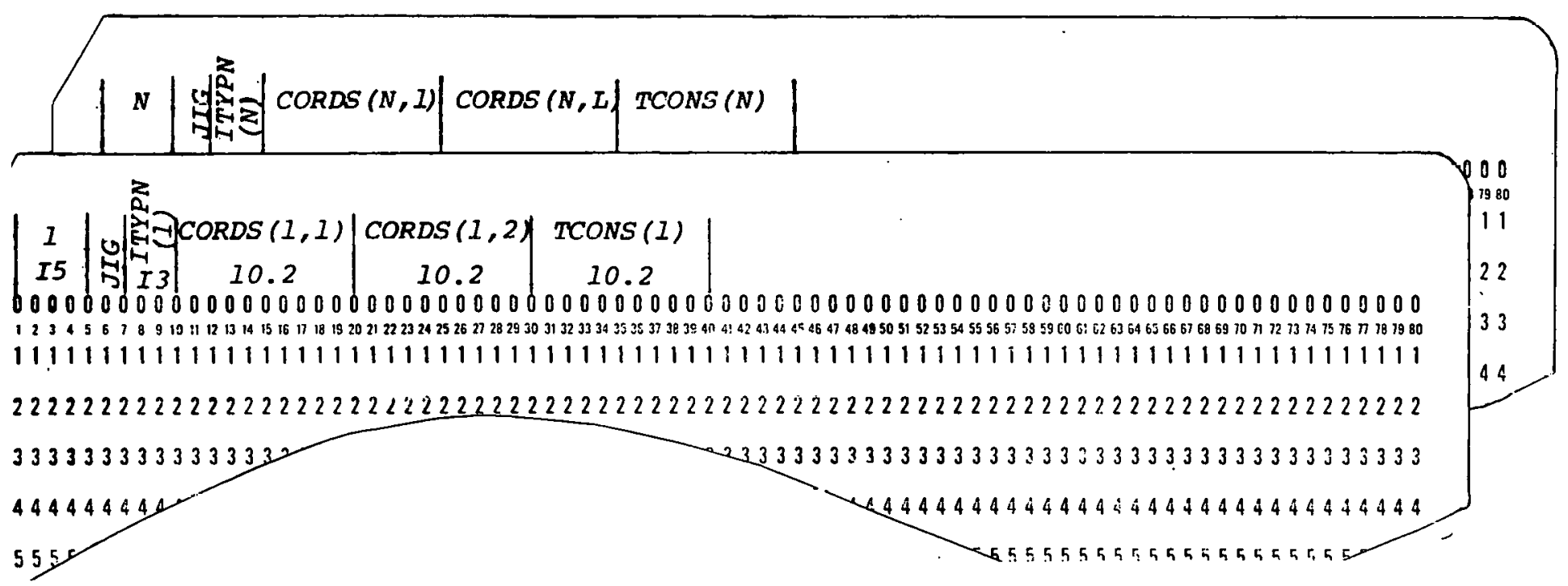


where:

$N$

$\operatorname{ITYPN}(N)$

JIG

$\operatorname{CORDS}(N, I)$

TCONS (N) is the nodal point number ( $n=1$, NNODES)

is a boundary condition code

$$
\begin{aligned}
\operatorname{IYTPN}(N) & =0 \text { for external heat flow specified } \\
& =1 \text { for specified temperature } \\
& =-1 \text { for initial temperature specified }
\end{aligned}
$$

controls generation of ITYPN and TCONS data for omitted nodal cords

$$
J I G=0 \text { results in ITYPN }=0 \text { and } \text { TCONS }=0
$$

for generated nodes

are the global nodal coordinates of node $N$ with

$\operatorname{CORDS}(N ; \mathcal{L})=X(N)$

$\operatorname{CORDS}(N, 2)^{\circ}=Y(N)$

is the constant temperature of node $N$ for ITYPN(N) $=1$. If a constant flux boundary is desired. TCONS represents the magnitude of the flux and ITYPN $(N)=0$ for nodes with specified flux.

DATA CARD (S) 9

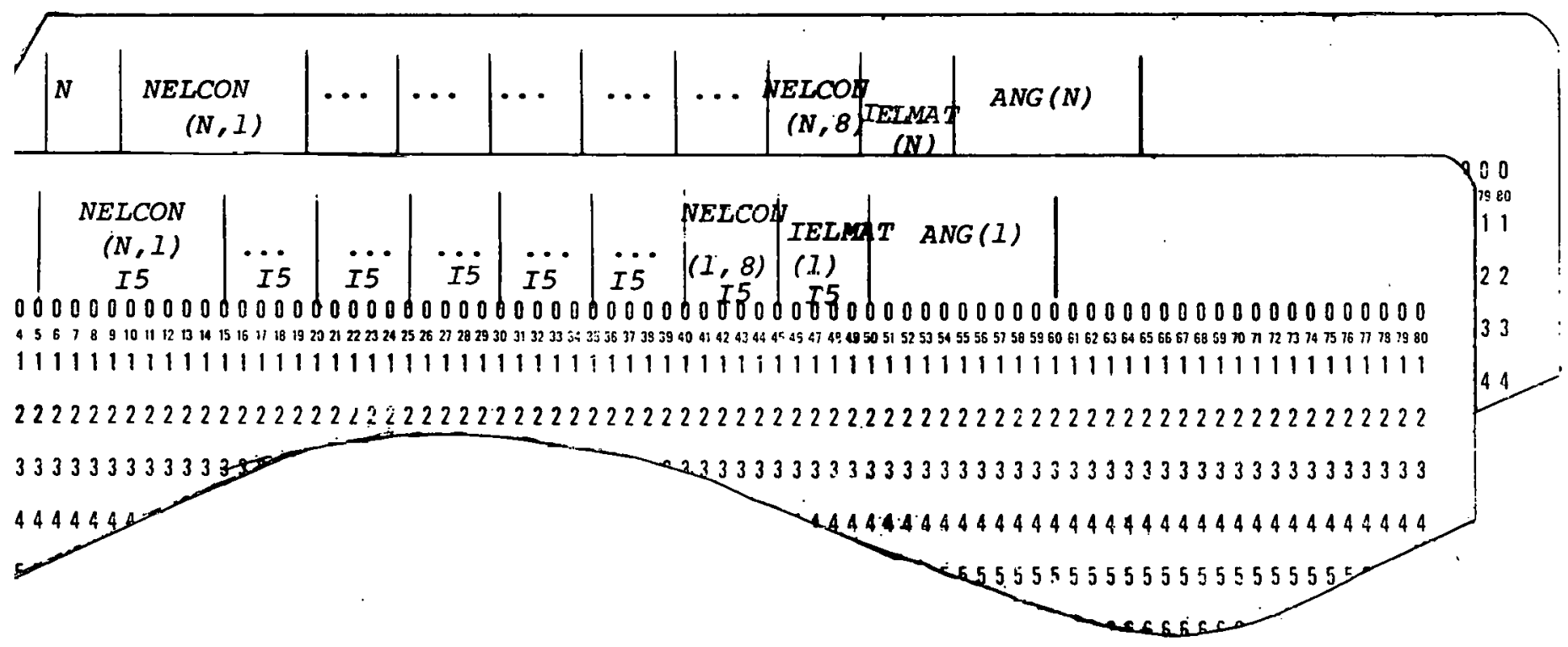


$N$ $\operatorname{NELCON}(N, I)-\operatorname{NELCON}(N, 8)$ is the element number $(N=1, N E L E M)$

are the global node numbers progressing counter clockwise in an element $N ; e . g .:$

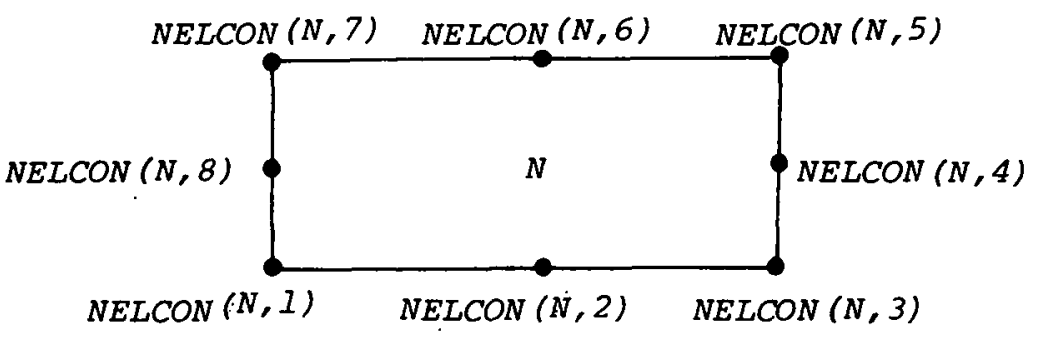

$\operatorname{IELMAT}(N)$

is the material type number of element $N$.

$A N G(N)$

is the angle of the principal conductivities from the global coordinate axes (counter clockwise is taken positive) for element $N$. If the conductivity matrix is supplied with respect to the global axes, the angle should be zero.

DATA CARD(S). 10

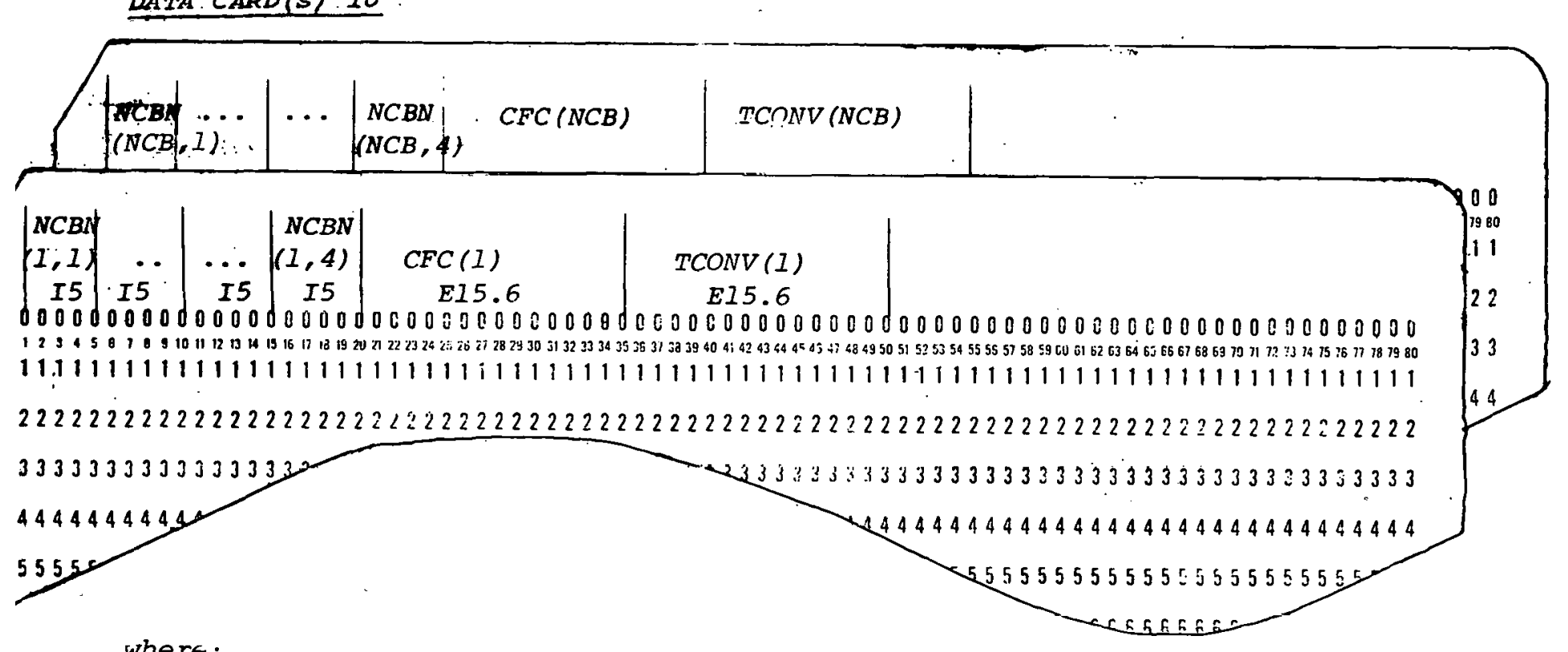

where:

$N C B N(N, 1)-N C B N(N, 3)$

$\operatorname{NCBN}(N, 4)$

$\operatorname{CFC}(N)$

$\operatorname{TCONV}(N)$ are the node numbers of the element on the convective boundary $(\operatorname{NCBN}(N, 2)$ is the secondary node)

is the number of the element on the convective boundary

the convective film coefficient $\left(M / t^{3} T\right)$

is the ambient air temperature; (T).

Omit data card(s) 10 if $N C \bar{B}=0$ (data card 4). 
Data card types 11-13 pertain to the initial temperature field data. Data card 11 is used in conjunction with either data card type 12 or 13 but not both.

DATA CARD 11

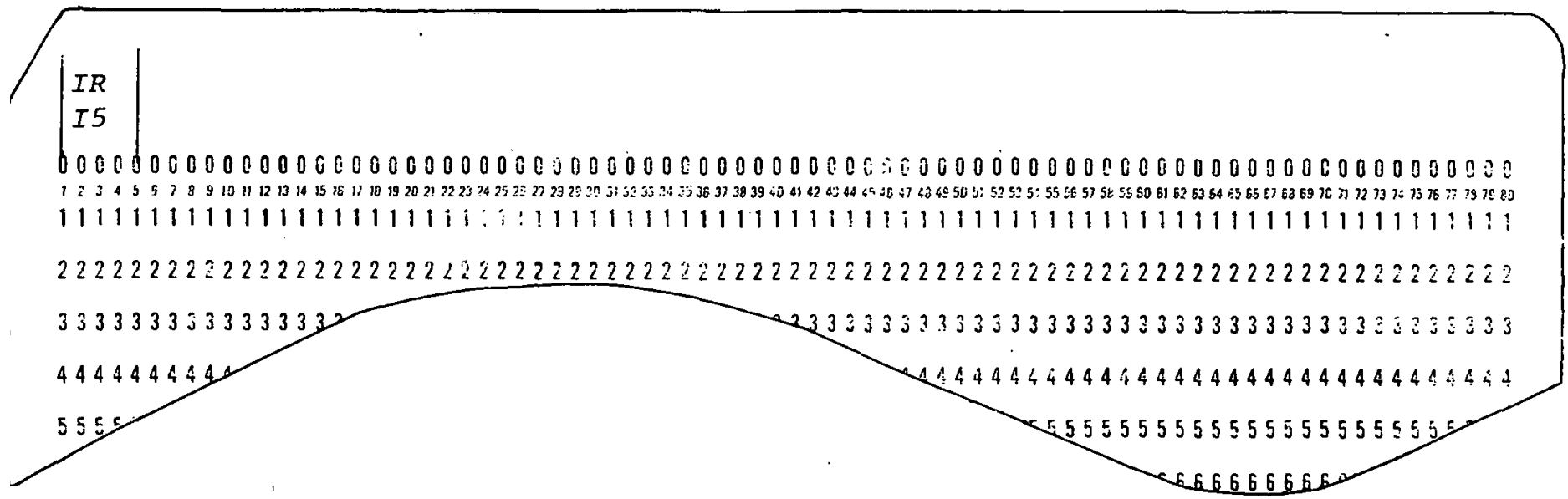

where:

$I R$

$$
\begin{aligned}
& \text { is a format control parameter. } \\
& I R=1 \quad \begin{array}{l}
\text { initial temperatures are read } \\
\text { in for every node }
\end{array} \\
& I R=0 \quad \begin{array}{l}
\text { Temperatures may be generated } \\
\text { between nodes, or temperatures } \\
\text { may be specified at particular } \\
\text { nodes }
\end{array}
\end{aligned}
$$

The $I R=1$ option is used together with data card type 12 that follows. This option requires that each nodal temperature be specified. For instance, if IWRIT $=2$ (data card 1), the temperature field at the final time-step (JTMAX) is punched according to the format specification for data card type 12. These punch cards could subsequently be used with $I R=1$ to restart the program at a later date. 
DATA CARD(S) 12

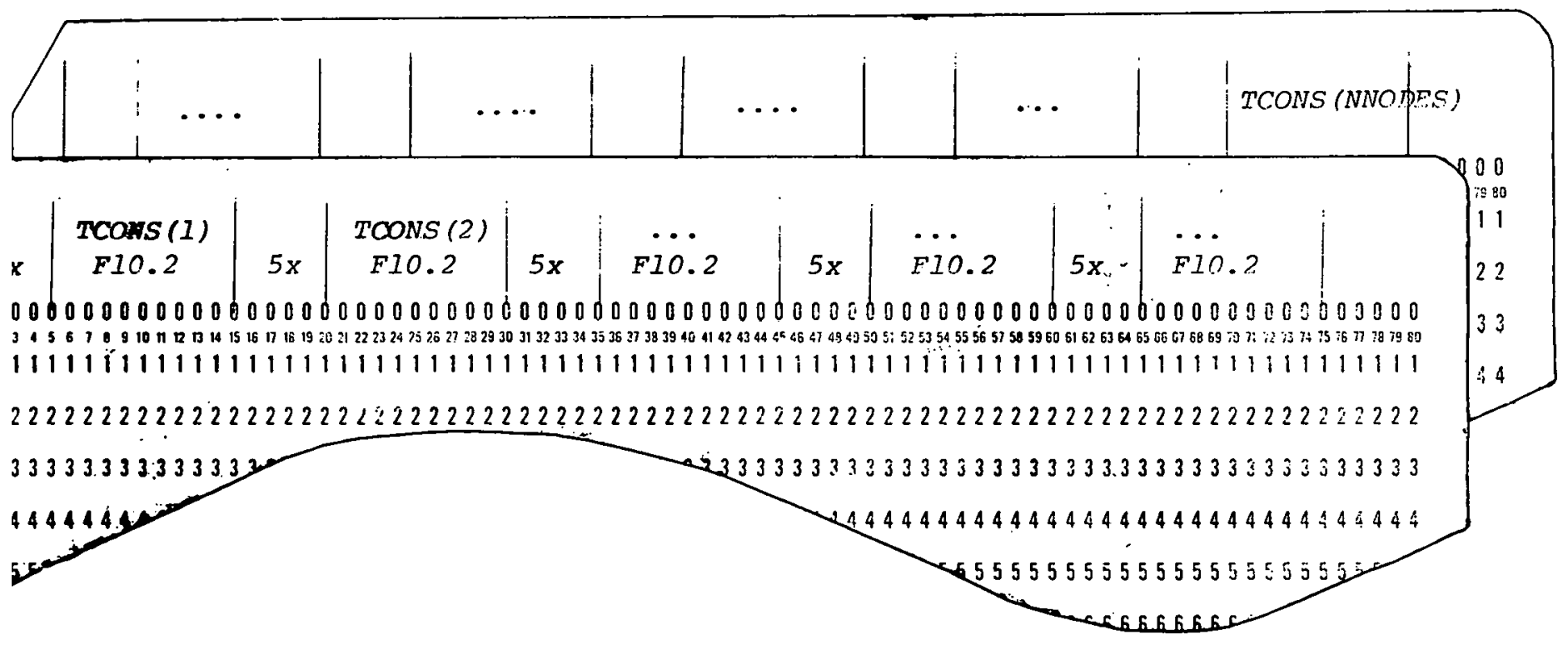

where:

TCONS(N) are the initial nodal temperatures.

Omit data card(s) 12 for IR $=0$ and for

steady-state problems (DELT $=0.0$,

data card 2)

DATA CARD(S) 13

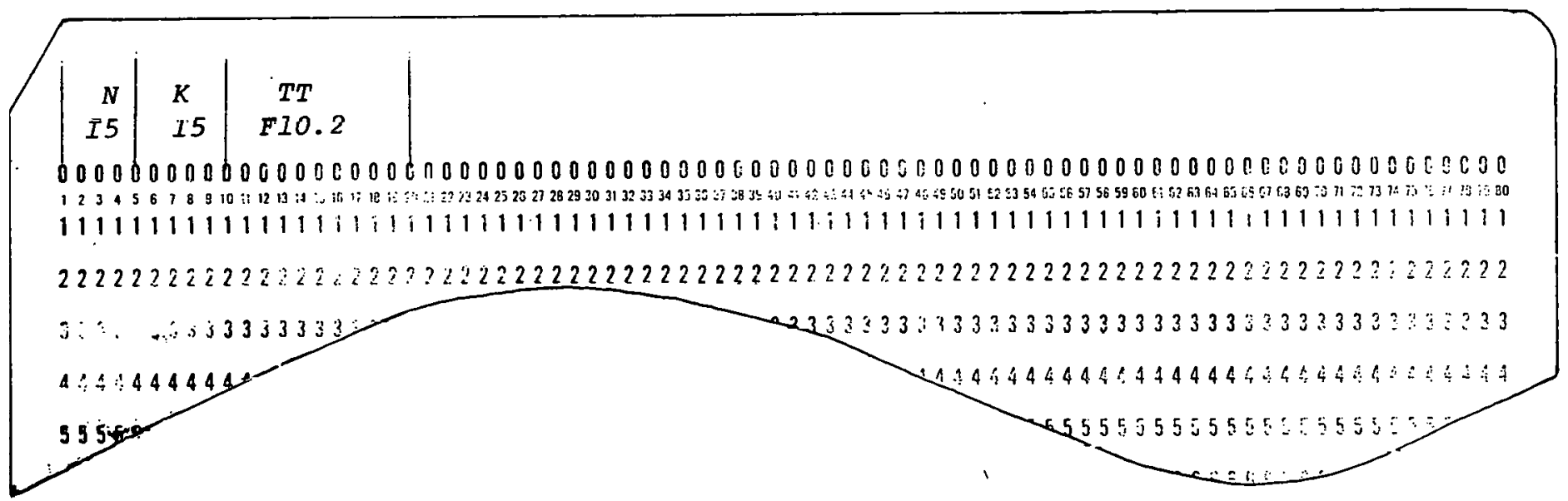

Data card type 13 is used if $I R=0$ (card type 11). Using card type 13 reduces data input since automatic generation is utilized where nodal temperatures are identical. 
End data card 13 sequence with one blank card unless the initial temperature field is zero. The following table illustrates the input procedure.

\begin{tabular}{|c|c|c|c|}
\hline$N$ & $K$ & $T T$ & Comments \\
\hline 0 & 0 & 0 & $\begin{array}{l}\text { All initial nodal temperatures are zero } \\
\text { except those specified as constant } \\
\text { temperature boundary nodes. No blank } \\
\text { end card required. }\end{array}$ \\
\hline$N I$ & 0 & $T T 1$ & $\begin{array}{l}\text { Nodes NI through NN are all initial- } \\
\text { ized to temperature TTl. }\end{array}$ \\
\hline NN & 1 & TTI & \\
\hline 0 & 0 & 0 & Blank end card. \\
\hline$N I$ & 0 & TTI & Nodes $N 1$ through $N N 1$ are all initial- \\
\hline$N N 1$ & 1 & $\operatorname{TT1}$ & $\begin{array}{l}\text { through NN2 are all initialized to } \\
\text { temperature TT2. Nodes not specified }\end{array}$ \\
\hline$N 2$ & 0 & $T T 2$ & $\begin{array}{l}\text { are taken to be zero and constant } \\
\text { temperature boundary nodes are }\end{array}$ \\
\hline NN2 & 1 & $T T 2$ & maintained. \\
\hline 0 & 0 & 0 & Blank end card. \\
\hline
\end{tabular}

Examples: Suppose we desire to give nodes 1 through 5 the temperature 100 and nodes 6 through 10 the temperature 200; then we would havce

\begin{tabular}{|r|c|c|c|}
\hline \multicolumn{1}{|c|}{$N$} & $K$ & $T T$ & \multicolumn{1}{|c|}{ Comment } \\
\hline 5 & 1 & 100 & $\begin{array}{l}\text { specifies noded } 1-5 \\
10\end{array}$ \\
1 & 200 & $\begin{array}{l}\text { specifies nodes 6-10 } \\
\text { end sequence card }\end{array}$ \\
\hline
\end{tabular}

Suppose we desire to give only nodes 5 and 10 the temperatures 100 and 200, respectively, then we would have: 


\begin{tabular}{|r|r|r|c|}
\hline \multicolumn{1}{|c|}{$N$} & $K$ & $T T$ & Comment \\
\hline 5 & 0 & 100 & Specifies just node 5 \\
10 & 0 & 200 & Specifies just node 10 \\
0 & 0 & 0 & End sequence card \\
\hline
\end{tabular}

Omit data card(s) 13 for $I R=1$ and for steady state problems $(D E L T=0.0$, data card 2).

\section{EXAMPLE PROBLEM}

\subsection{Problem Description}

Consider heat conduction in a slab of width $2 L$, initially at uniform temperature $T_{i}$. For times $t>0$, both boundaries $(x= \pm L)$ are kept at constant temperature $T_{b}$ as depicted in Figure 2 .

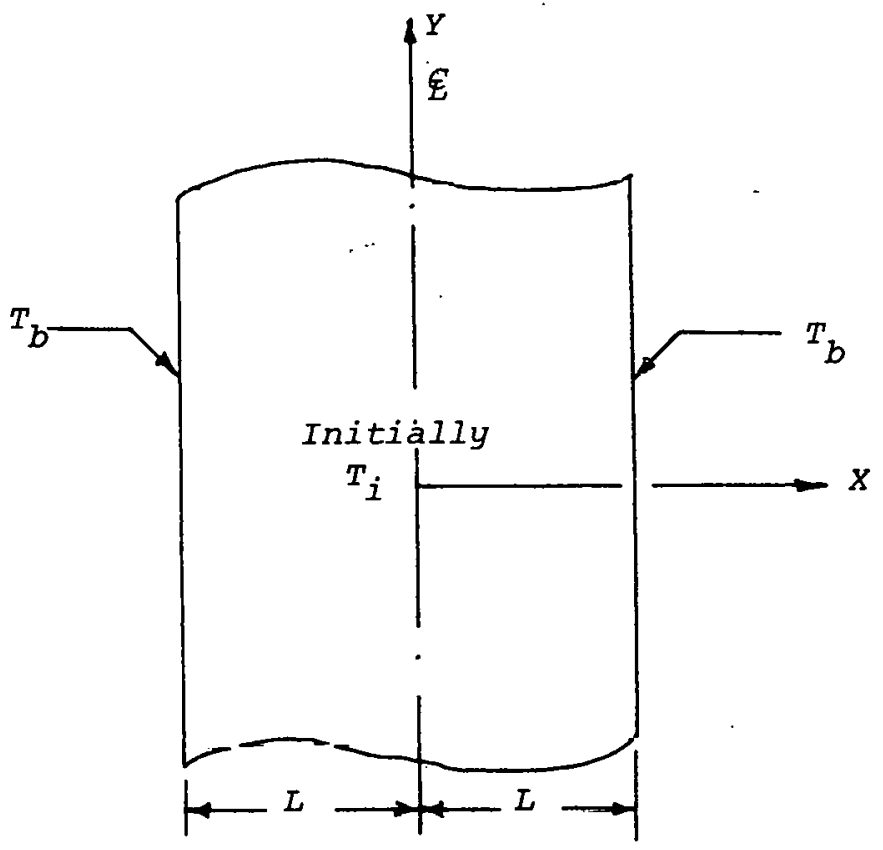

Figure 2. Example problem geometry

The above problem is presented by Ozisik (3) who utilized the numerical results of Dowty and Haworth (4) where the thermal conductivity of the material varied linearly with temperature, viz

$$
k=k_{0}(I+\beta T)
$$


where:

$$
\begin{aligned}
& k_{0}=\text { thermal conductivity for temperature of zero degrees. } \\
& k_{0} B=\begin{array}{c}
\text { temperature coefficient of conductivity (SLOPE } \\
\text { parameter) }
\end{array} \\
& T=\text { temperature at time } t . \\
& \text { Results are presented graphically for centerline temperatures }\left(T_{C}\right)
\end{aligned}
$$
by Ozisik (3) in terms of dimensionless temperature, $\theta$, and dimensionless times, $\tau$, for various values of $\psi$ with

$$
\begin{aligned}
\theta=\left(I+0.4 \beta T_{i}\right) \frac{T_{C}-T_{b}}{T_{i}-T_{b}} \\
\tau=\frac{\alpha_{0} t}{L^{2}}, \quad c_{0}=\text { initial thermal diffusivity } \\
t=t i m e \\
L=s l a b \text { half width } \\
\psi=10 B T_{b}
\end{aligned}
$$

Choosing the initial and boundary conditions as:

$$
\begin{aligned}
& T_{i}=1000_{F} \\
& T_{b}=500^{\circ} F
\end{aligned}
$$

and the following values for material parameters;

initial thermal conductivity, $k_{0}=1.0 \frac{B t u}{\mathrm{Ft}-\mathrm{hr}-{ }_{\mathrm{F}}}$

temperature coefficient of thermal conductivity, $B=1.6 \times 10^{-3} / o_{F}$

density: $\rho=1001 b_{m} / f t^{3}$

specific heat: $C_{p}=0.1 \frac{B t u}{1 b_{m}^{-O_{F}}}$

initial thermal diffusivity: $\alpha_{0}=0.1 \mathrm{ft}^{2} / \mathrm{hr}$

slab half width: $L=1 \mathrm{ft}$

results in the following expressions for centerline temperature and time for a fixed value of $\psi$ : 


$$
\begin{gathered}
{ }^{T}{ }_{C}=500-375.94 \theta \\
t=10 \tau \\
\psi=8.0
\end{gathered}
$$

Extrapolating several points from the plot presented by Ozisik we obtain:

\begin{tabular}{|c|c|c|c|}
\hline$\tau$ & $t$ (hrs.) & $\theta$ & $T_{C}\left(\rho_{F}\right)$ \\
\hline 0.4 & 4 & 0.3 & 387 \\
0.5 & 5 & 0.2 & 425 \\
1.0 & 10 & 0.02 & 493 \\
1.5 & 15 & 0.002 & 499 \\
\hline
\end{tabular}

\subsection{Finite Element Discretization}

In modeling the slab problem, the characteristic height of the finite element model is arbitrary and $0.25 \mathrm{ft}$. is chosen. The slab is symmetric about the $y$-axis, thus a section $0.25 \mathrm{ft}$. high with a slab half width of one foot is chosen.

The mesh is shown with the appropriate boundary conditions in Figure 3. The circled numbers represent the element numbers with the remainder representing the nodal numbers.

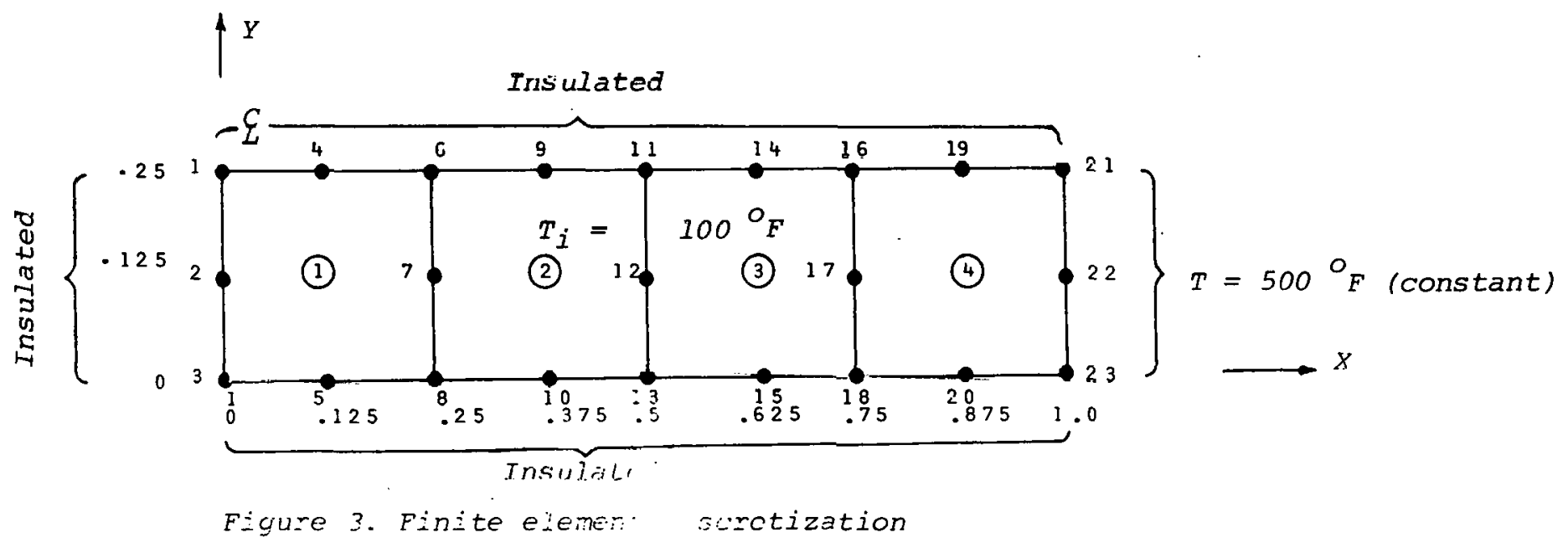




\author{
3.3 Input Data \\ After the preliminary items regarding a specific problem \\ (as completed in section 3.1 and 3.2) have been finalized, one may \\ proceed to describe the problem for adaption to the computer program. This \\ has been done following the procedure of data input described in \\ section 2 and is illustrated by the coding forms on the following pages.
}


IBM

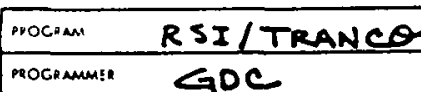

gORTRAN Coding Form

Donit
Qx28.7327.6 U/M 050. rinted in U.S.A.

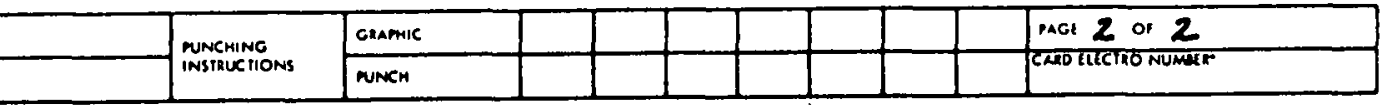

FORTRAN STATEMENI

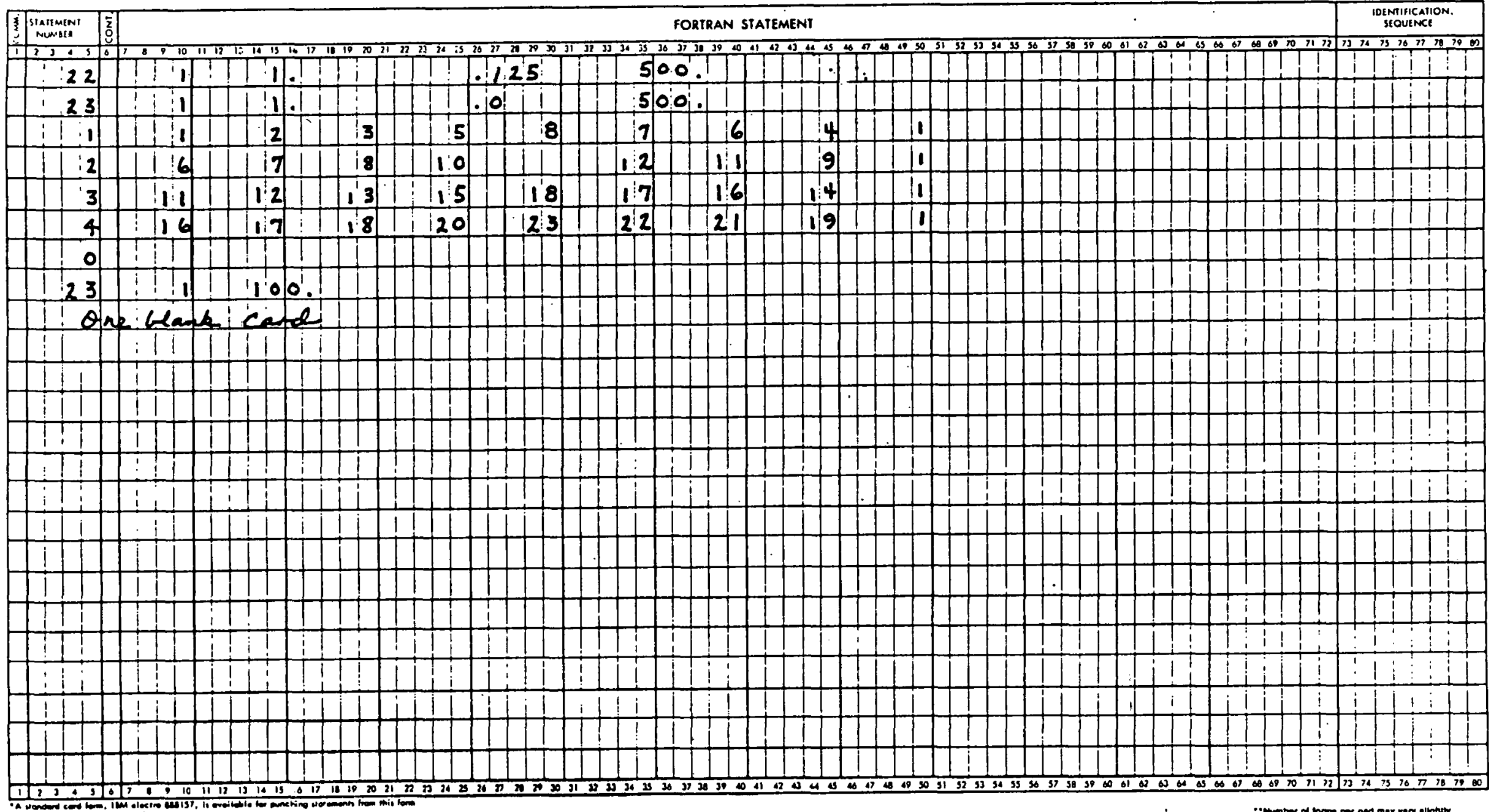




\subsection{Results}

The following pages illustrate the output from the computer

code and the results of the example problem including the input

data and the nodal temperatures for each time step. Nodes 1, 2, and 3

represent the center line of the slab. Comparing these temperatures

with those computed in section 3.1 for times $t=4,5,10$, and 15 hrs., one observes that the two solutions agree within a few percent. 
RSI/IKANCO EXAMPLE PROBLEM HITH..LINEAR JEMPEKATURE DEP ...CONOUGTIVITIES

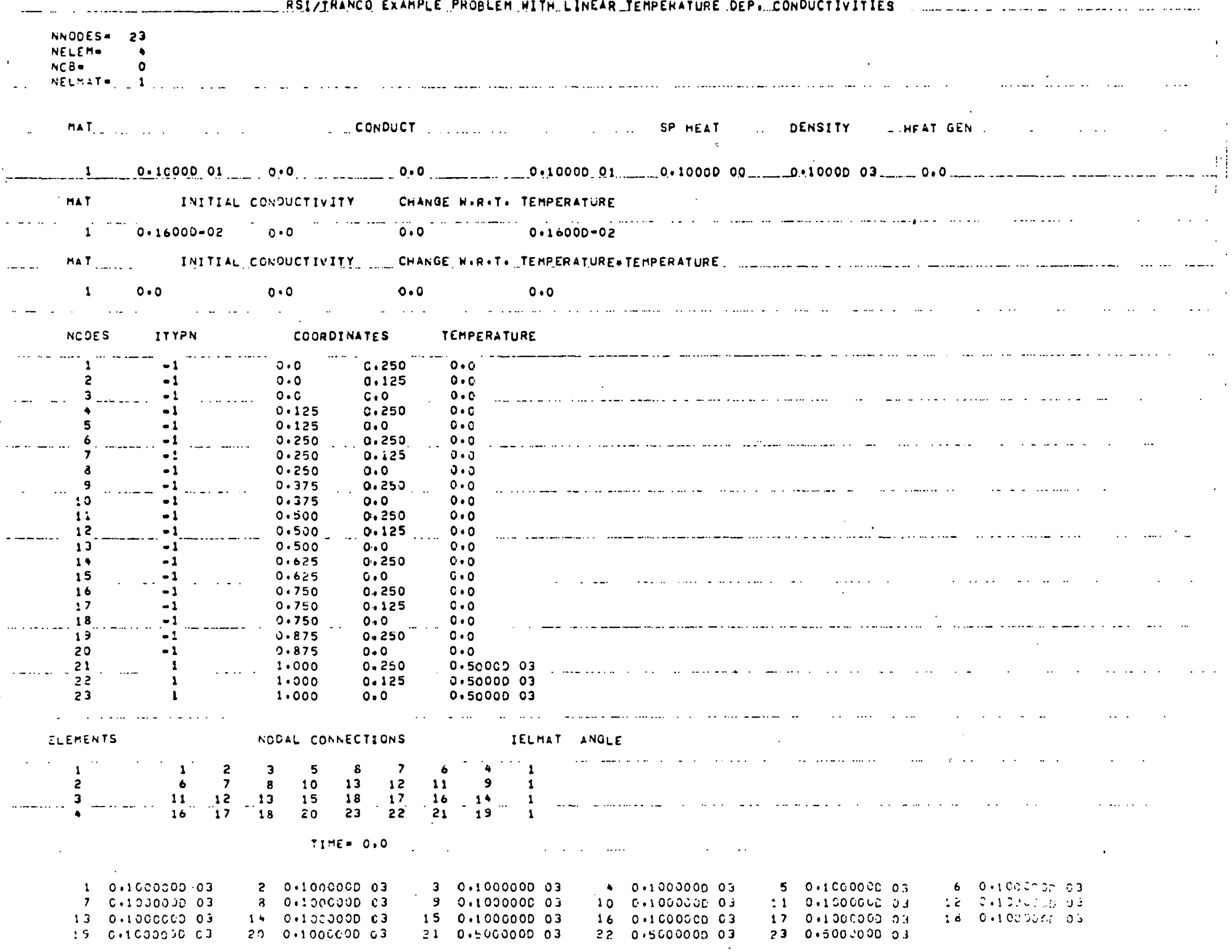




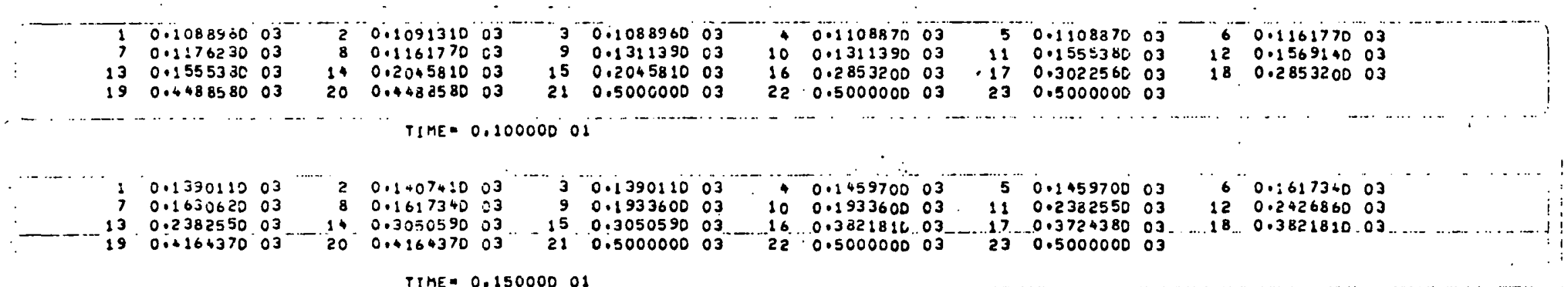

TIME $=0.15000001$

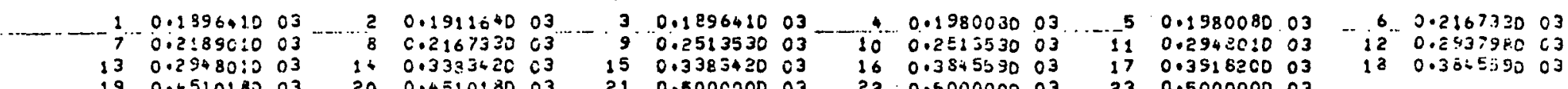

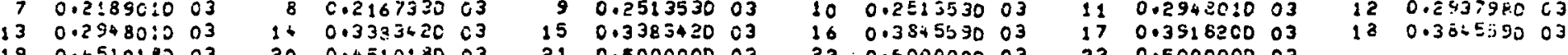

TIME $=0.20000001$

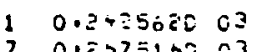

$0 . \overline{0} 57510023$

190.453539003

20000001

$20.2+.257003$

$\therefore 0.265604003$

20.053589003

\section{2 .2562003 \\ 9. 0.293630003}

15.368756003

$+0.250272003$

10. 0.253650003

$160.41644+003$

TIME $=0.25000001^{-}$ $\begin{array}{lll}2 & 0.29026+0 & 03 \\ 8 & 0.3975190 & 03\end{array}$ 0.390005003

$20 \quad 0.663431 C_{03}$

\subsection{3 0.330410003} 15
21
21 0.35000000503
0.294979003 100.330410003

220.500000003
$5 \quad 0.250272003$

11. 0.326721003

170.433042003

230.500000003
60.265654003 $120.3=5940003$ $180.41 t+9.9003$

TIME $=0.300000 .01$

0.3277110 .03 $0.36350=003$ $0.3825+5203$ is. $0.06397 \times 003$
2. 0.328733003 $80.342551003 \quad 0.360655003$ 100.4097980 c3 150.4097380 03 $210.5000000 \mathrm{~J}$
4.0 .332519003 $100.3600550 \mathrm{c}$ 160.440165003 $22.0 .5000000 \mathrm{c} 3$
50.294979003 110.359001003 230.500000003

$\begin{array}{rrr}6 & 0.3075190 & 03 \\ 12 & 0.3589290 & 03 \\ 18 & 0.4242270 & 03\end{array}$
$\begin{array}{lll}12 & 0.3589290 & 03 \\ 18 & 0.4242270 & 03\end{array}$

TIME $=0.35000001$

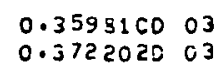
$0.3722020 \mathrm{cos}$ $\begin{array}{lll}0.404 i 680 & 03 \\ 0.47+50 \leqslant 0 & 03\end{array}$

\subsection{3 $0.3714530 \quad 03$} 0.4256530
0.4745060
0.3
$0.3598100 \quad 03$

$0.386011003 \quad 100.363470003$ $150.625053003 . . .100 .0 .306011003$ $210.500000003 \quad 220.500000003$

- ... TIME* 0.40000001

5.0 .3325190 .03 $\begin{array}{lll}11 & 0.3325400 & 03 \\ 17 & 0.4393510 & 03\end{array}$ 23... 0.500006003
$6-0.3428510 \quad 03$ $\begin{array}{lll}12 & 0.3335450 & 03 \\ 18 & 0.440: 650 & 03\end{array}$

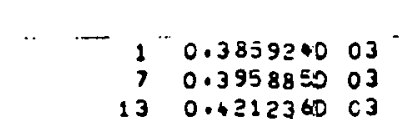




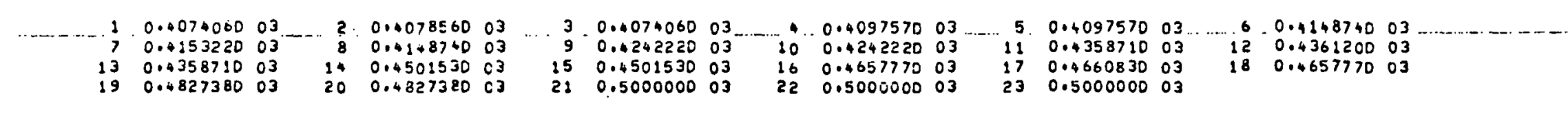

TIME 0.50000001 .

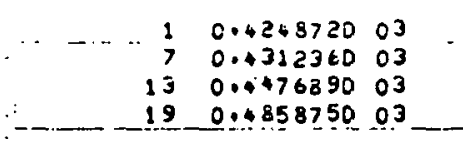

$\begin{array}{rll}2 & 0.42525+0 & 03 \\ 8 & 0.4309090 & 03 \\ 14 & 0.4593510 & 03\end{array}$

$30.4 \bar{c} 4272003$ 50.459351003 $\begin{array}{lll}10 & 0.4383920 & 03 \\ 16 & 0.4732220 & 03\end{array}$ $0.4858750 \quad 0.3$ 0.00452520
50.426777003 110.4476890 .03
$6 \quad 0.43090 .50 \quad 03$ $120.4479700 \quad 03$

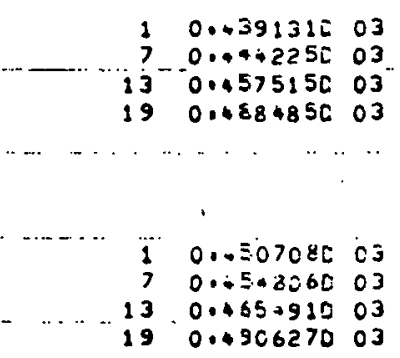

190.0906503

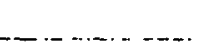

$\begin{array}{rlll}\ldots \ldots . .1 & 1 & 0.4501170 & 03 \\ & 7 & 0.0634090 & 03 \\ 13 & 0.4720300 & 03\end{array}$

$\begin{array}{lll}13 & 0.4720360 & 03 \\ 19 & 0.4923850 & 03\end{array}$

…………

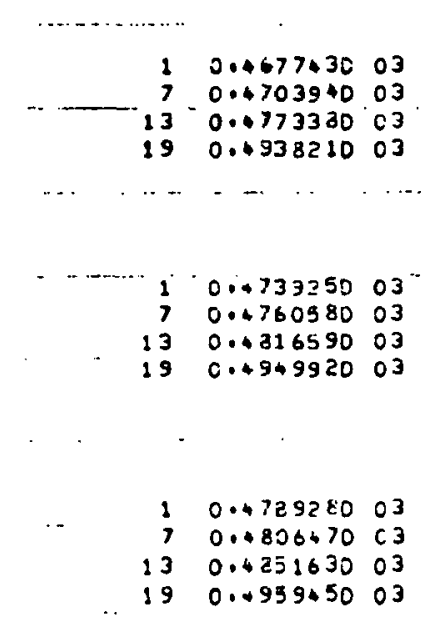

TIME- 0.55000001

\section{$0 .+39+26003$ $0.4+39450 \quad 03$}

0.1000918003

30.439131003 0.440647003 $150.466918003 \quad 16-0.477249003$ 10.500000003 330.500000003 $\begin{array}{llllll}16 & 0.4772490 & 03 & 17 & 0.4773930 & 03 \\ 22 & 0.5009000 & 03 & 23 & 0.5000000 & 03\end{array}$

TIME $=0.00000001$

$\begin{array}{lll}2 & 0.4505500 & 03 \\ 8 & 0.4545890 & 03 \\ 14 & 0.4731220 & 03\end{array}$

14 0.4731220 0j

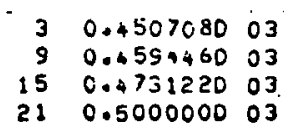

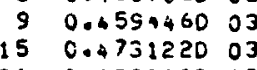

10.500000003
$0.4406470 \quad 03$

$\begin{array}{llll} & 0.4439450 & 03\end{array}$ $\begin{array}{llll} & \\ 0773930 & 03 & -12 & 0.4577032 .03 \\ 0 & 0.4772+9503\end{array}$

TIME $0.85000001 \ldots$

0.460309003 0.463230003 $0 .+7819100$

$30.460: 170 \quad 03$ 0.492385003

TIME 0.70000001

0.467899003 0.470252003 0.4823240 03 0.493821003

\section{TIME $=0.75000001$}

\section{$30.467743003 \quad 0.468534003$ 0.482324003 10.500000003 0.468534003 10.0 .473407003 $22 \quad 0.500000003$} $11 \ldots 0.477334003$ 170.487875003 230.500000003

50.401097003 230.500000003

$\therefore \quad 6.0 .463230003$

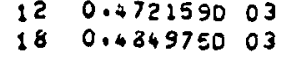

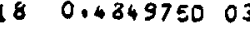

$0.4740500 \quad 03$ $0.4759430 \quad 03$ 0.475963003

3 . 0.473925003 $\begin{array}{lll}3 & 0.4739250 & 03 \\ 9 & 0.4784870 & 03 \\ 5 & 0.4556860 & 03\end{array}$

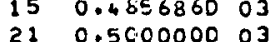

$\begin{array}{rlr}4 & 0.4745600 & 03 \\ 10 & 0.4784870 & 03\end{array}$ $0.47+560003$ 200.485866003

TIME 0.80000001

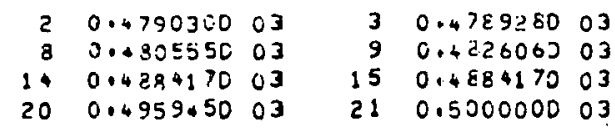

$4 \quad 0.475441003$
$10 \quad 0.482606003$
$16 \quad 0.492006003$ 100.482606003 560.590000003 
$13 \quad 0.4880040 \quad 03$ $-\frac{1}{20}-\cdots 0.004$

$0.490632033 \ldots 15 \ldots 0.4906320 \ldots 3$

$16-0.0493530003$

$\begin{array}{lll}17 & 0.4935610 & 03 \\ 23 & 0.5000000 & 03\end{array}-18-0.0493530003$

TIME 0.90000002

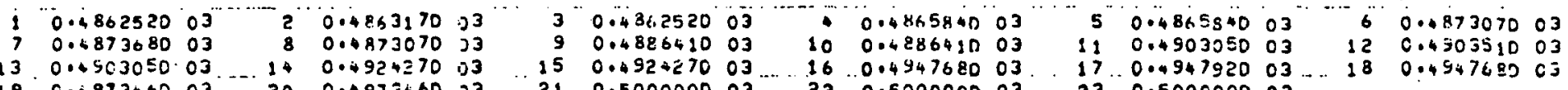

$13 \cdot 0.45630 \leq 0.03 \ldots . .140 .4924 \leq 7003$

$\begin{array}{lllllll}15 & 0.492+\angle 70 & 03 & \ldots .16 & 0.164947680 & 03\end{array}$

$230.5000000 \quad 03$

TIME $=0.95000001$

10.483898003

70.469798003

$20.4839510 \quad 03$

0.4897490 .03

$\begin{array}{llll}3 & 0.4888982 & 03\end{array}$

150.493830003

40.489166003

140.693880003

21 0.500000003

$\begin{array}{lll}0 & 0.4908250 & 03\end{array}$

$6.4957700 \quad 03$

$5.0 .4891660 \quad 03$

110.492167003

$\begin{array}{lll}1 & 0.4957900 & 03 \\ 2 & 0.5000000 & 03\end{array}$

$60.4897450 \mathrm{C3}$

TIME $=0.10000002$

TIME $=0.1000000$

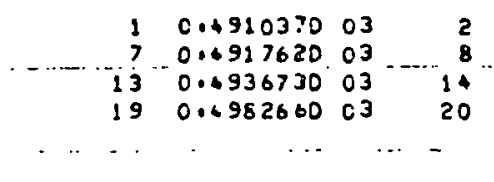

$\begin{array}{ll}0.4910790 & 03 \\ 0.4917230 & 03\end{array}$

0.495050003

$30.49: 0370 \quad 03$

9.0 .492591003

$15 \cdots 0.455056003$

40.491253003 0.492591003 0.490582003

$5 \quad 0.4912530 \quad 03$

$\begin{array}{llllll}530 & 03 \quad 6 & 0.4917230 & 03\end{array}$

" TIMEN 0,10500002

220.500000003

0.4965580 .03

120.493703003

0.500000003

8.496332003

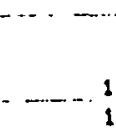

$0,+927640^{-} 03^{\circ}$ $0.493369003 \quad 20.492793003$

80.493317003

$\begin{array}{lllll}10 & 03 \ldots 14 & 0.49600 .70 & 03\end{array}$

$\begin{array}{lll}3 & 0.4927640 & 03 \\ 9 & 0.4940180 & 03 \\ 15 & 0.4560070 & 03 \\ 21 & 0.5000000 & 03\end{array}$

$\begin{array}{lll}4 & 0.4929330 & 03 \\ 10 & 0.454018003 \\ 16 & 0.4972390 .03\end{array}$

5 $0.4929380 \quad 03$ 0.49335700703
0.496599003

$\begin{array}{ll}16 & 0.4972390 .03 \\ 22 & 0.500000003\end{array}$

$10.4948910 \quad 03$

7. 0.497252003

TIME 0.11000002

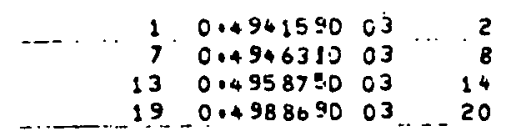

$\begin{array}{lrlll}0.4941870 & c 3 & 3 & 0.4941590 & 03 \\ 0.4946060 & 03 & 9 & 0.4951700 & 03 \\ 0.4967760 & 03 & 15 & 0.4567760 & 03\end{array}$

$0.04943000 \quad 03$ $0.5000000 \quad 03$

6 0.493317003

120.496915003 180.4972390 .03

50.494300003

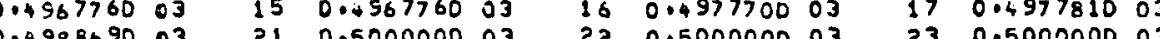

TIHE $=0.11500002$

$\begin{array}{llll}1 & 0.4952860 & 03\end{array}$ 0.4956660003 $0 .+95676000$

$20.4953080 \quad 03$ $0.4956460 \quad 03$ 0.497397003

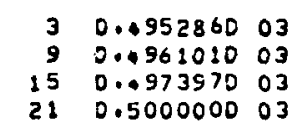

0.0495399003 $0.49987003 \quad 21 \quad 0.500000003 \quad 160.4981950 \mathrm{c3}$ 110.496670003

TIME $=0.12000002$

$10.496195003^{-1} 20.496213003$ $7 \quad 0.19650=0 \quad 03$ 80.496486003 $\ldots . .13-0.49731=0 \quad 03$ $14-0.4978990$
20
0.04592630 03

$30.096 \pm 95003$ 40.495287003

TIME= 0.125000 .

$0.4962870 \quad 03$ 70.493503 230.500000003 


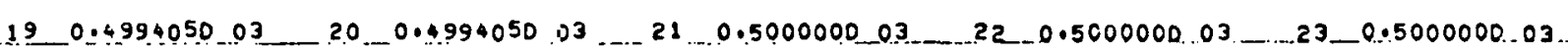

PIME- 0.13000002

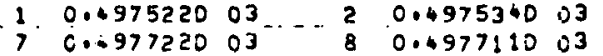

$13 \quad 0.4952490 \quad 03 \quad: 40.4986310 \quad 03$

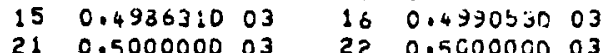

5.0 .497582003

110.498249003

60.4977110 .03

TIME $=0.13500002$

10.498001003

7.0 .498162003

$130.49858: 003$

0.698010003

0.498153003

0.498895003

$20 \quad 0.4996120 \quad 03$

TIME $=0.14000002$

70.498517003

$130.4988600 \quad 03$

$19.0,499887003$
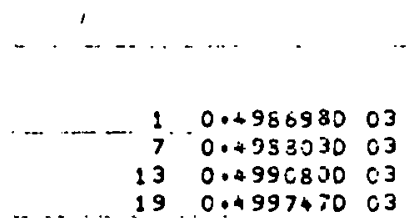

$130.499 C 85003$

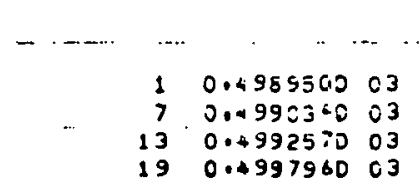

0.0203003

$0.4997960 \quad 03$

$0.6991520^{\circ} 03$ 0.299221003 130.099401 .003

13.0 .499401 .003

\section{0.499156003 \\ 0.699217003}

20.0065935003

TIME $=0.16000002$ $\begin{array}{llll}1 & 0.6993360 & 03 \\ 7 & 0.09937: 0 & 03\end{array}$ 130.499516003

$0.4993190 \quad 03$ 0.4993680 .03 140.4996220 03 $20 \quad 0.4998670 \mathrm{J3}$ 0.495434003

\section{TIHE= $0.165000 \quad 02$}

TIME 0.14500002 0.4903323003

TIME $=0.15000002$

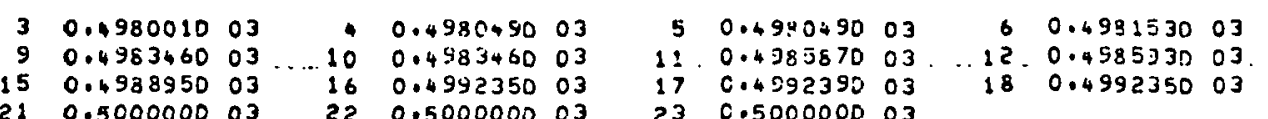

$23 \quad 0.4992390 \quad 03$

80.499235003
0.498510003
$3 \quad c .498387003$
$\begin{array}{lll}5 & 0.4984250 & 03 \\ 1 & 0.4933500 & 03\end{array}$
6 $0.4905100 \quad 03$
$16 \ldots .0 .4593830 \quad 03$
170.493385003
20.498555003
$18 \quad 0.4593830 \quad 03$

0.499687003

2) 0.500000003

230.500000003 $\begin{array}{llllll}0.4987040 & 03 & 3 & 0.4986980 & 03 \\ 0.4987970 & 03 & 9 & 0.4929330 & 03\end{array}$ 210.500000003

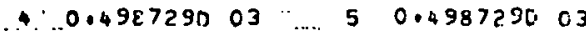
$\begin{array}{lllllll}10 & 0.4969230 & 03 & 11 & 0.4950800 & 03\end{array}$ $160.499502003 \quad 170.435504503$ $0.500000003 \quad 230.500000003$
6. 0.498797003

$12 \quad 0.49904 \div 003$

180.439552003
$4 \quad c .498975003$ $10 \ldots .0 .499: 310 \quad 03$ 16.10 .499598003 $0.5000000 \quad 03$

$5 \quad 0.49897=0 \quad 03$ 0.499257003 $0.4996000 \quad 03$ 0.500000003

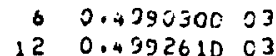
180.499598003 
TIME - 0.170000 .02

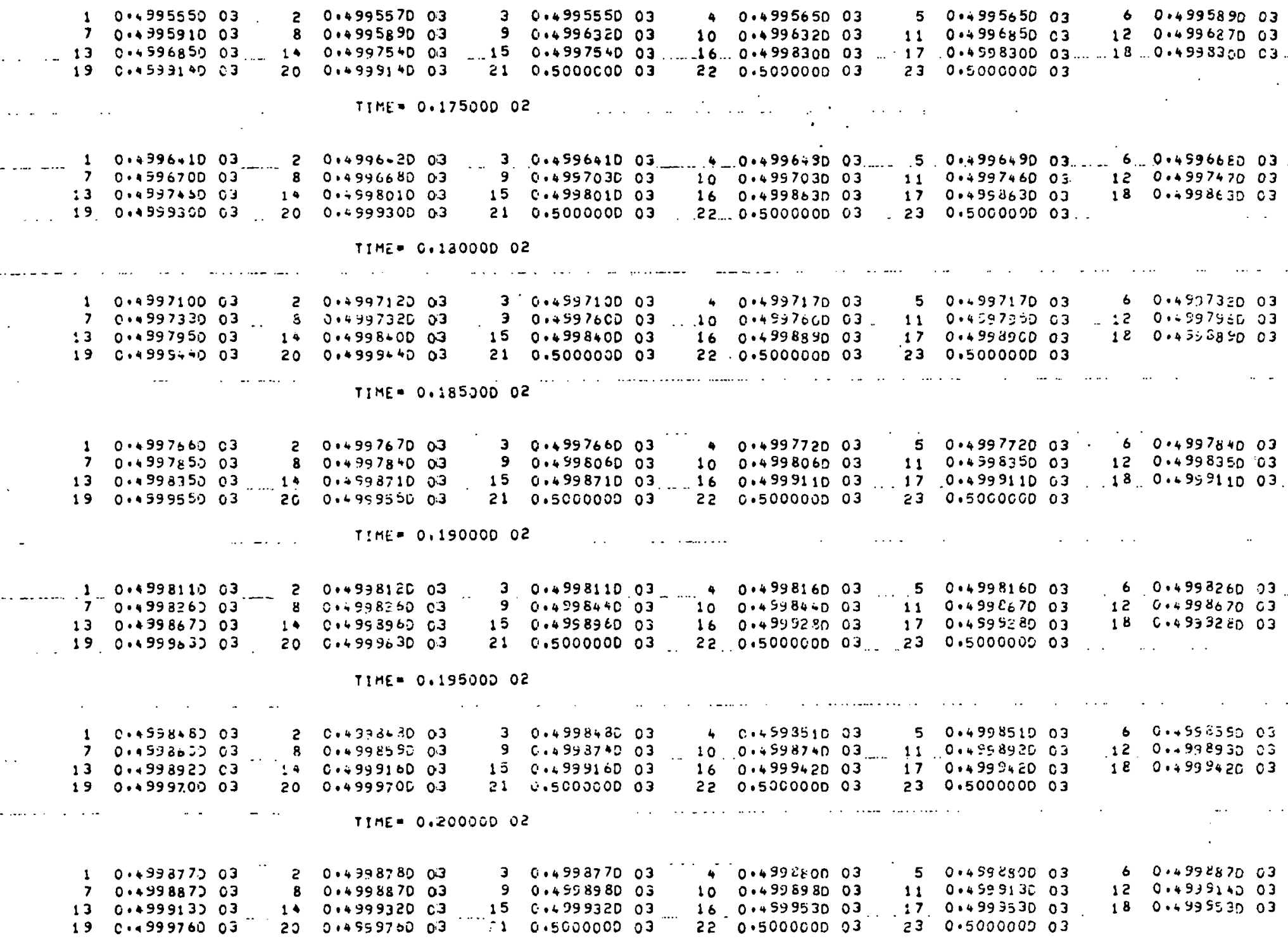

IHCONZI STIP 
1. Zienkiewicz, O.C., The Finite Element Method in Engineering Science, MCGraw-Hili, 1971.

2. Desai, C.S., and Abe1, J.F., Introduction to the Finite Element Method, A Numerical Method for Engineering Science, Van Nostrand Reinhold Company, 1972.

3. Ozisik, M.N., Boundary Value'Problems of Heat Conduction, International Textbook Co., 1968, pp. 377-381.

4. Dowty, E.L. and Haworth, D.R., "Solution Charts for Heat Conduction in Materials with Variable Thermal Conductivity," ASME Paper 65-WA/H'l-29, 1965. 


\title{
APPENDIX A
}

\section{Fortran Listing of Computer Code RSI/TRANCO}

\author{
Included in the following pages is a fortran listing of computer code \\ RSI/TRANCO. With the current dimensions, the program may be run as a \\ Class $C$ JOb on the ORNL IBM 360/91 system. \\ Program limits are as follows:
}

\begin{tabular}{lr}
\multicolumn{1}{c}{ ITEM } & MAXIMUM \\
Number of Nodes & 1500 \\
Number of Elements & 310 \\
Number of Different \\
$\quad$ Material Types \\
$\begin{array}{l}\text { Number of Convective } \\
\text { Boundaries } \\
\text { Maximum Semi-Band width }\end{array}$
\end{tabular}


:PILER OPTIONS - NAME = MAIN,OHT=OZ, LINECNT =60, SIZE= ODOOK, SOUHCE, EBCOIC, NOLIST, NODECK, LOAD, MAP, NOFUIT, NRIIL, NUXREF

C PROGRAM RSI TRANCO

$\mathrm{C}$

TRANCO SOLVES TRANSIENT OR STEADY STATE HFAT ....... ....... CONEUCTION PROBLEMS WITH FIXED TEMPFRATURE BOIINDARIES OR • C ........ CONVECTIVE BOUNDARIFS WHERE HEAT TRANSFER MAY BE .......

$C$
$C$
$C$
$C$
$C$
$C$
$C$
$C$
$C$
$C$
$C$
$C$
$C$
$C$
$C$
$C$
$C$
$C$
$C$
$C$
$C$
$C$
$C$
$C$
$C$
$C$
$C$
$C$
$C$
$C$

$\mathrm{C}$

C

C

$c$

C

C

c

1 READ $(5,500, E N D=2)$ NPROB, JTMAX, I J, IWRIT,MIT

IMPLICIT REAL*8(A-H,O-Z) INTEGER II-N)

COMMON BEOMTX(240,120), HEAT(1500), TOL, MBAND, MTT

COMMON/TVES, COROS $(1500,2)$, NELCON(310,8), IEL MAT(310)

COMMON/TVE/ NELFM, NELMAT, NNODES

COMMON /DELCI,M/ DELT, TM,JT, JTMAX, IPR

COMMON/PROF, CUND(4,2, 2$), C P(4), D E N(4)$, GHGEN(4)

1 , SLOPE $14,2,2)$, SLOP2 $(4,2,2), A N G(310)$

COMMON/TBOUN, CFC(50), TCONV $(50)$, TCONS $(1500)$, NCB, NCBN(50,4),

1 I TYPN (150E)

COMMON/HAI, $\quad A(8), Q G(8)$

COMMON /VAFI / IAMT, JTROL, JCNT, ITDEP

32 READ 51 , LELT, IPR, ITDEP

C

CALL INPUT

$J T=1$

$J J=1$

$T M=0 \cdot 0$

IFINIT ELT D O) GO TO 15
110

40

50

60

90

140

150

160

170

180

190

?. 00

260

270

280

290

300

310

320

410

430

490

500

530

540

550

8670 
FUF $K$. UT - O TEMPS ARE GFNERATEU BFTWEEN NOOFS

-..- FND SEqUENCE WITH CINE HLANK T.ARD

ENTER BLANK CARD FOR ZERO INTTIAL TEMPFRATURE

FOR TIRECT SOLUTIUN DO NOT READ IN

INTTIAL TEMPS FOK CONSTANT TEMP BOUNOARIFS

OR KEAD THEM IN AS ZFRO

C

C

C

C

C

$$
\begin{aligned}
& \text { READ INITIAL TEMP TYPE FORMAT } \\
& T R=1 \quad 5(5 x, F 10.2) \\
& T R=0 \quad(2(5, F 10.2) \text { AND GENERATE }
\end{aligned}
$$

8730

8740

8750

8760

8770

8780

READ 3?0, IR

320 FORMATII5)

IF(IR.ED.O) GO TO 321

8790

RFAD 32E; (TCONSIN),N=1, NNODES)

322 FORMAT $1515 \%, F 10.211$

GO TO 323

$321 N N=0$

922 READ 9? N, NoK,TT

IF (N.LE.O) GO TO 924

8810

8820

IF $(K \cdot L E \cdot O)$ GO TO 292

$I=N N+1$

$J=\mathrm{N}-1$

DO $293 \mathrm{~L}=\mathrm{T}$

$293 T C O N S(L)=T T$

292 TCONS $(N)=T T$

$N N=N$

GO TO 922

921 FORMATI 2 I5SF 10.2$)$

C

924 CONT INUE

8840

8850

8860

8880

8890

8900

8910

8920

8930

8940

8950

8960

8970

8980

8990

PRINT INITIAL NODAL TEMPERATURES

9000

C

323 PRINT 513,IM

PRINT $514 ;(N, T C O N S(N), N=1, N N O D E S)$

c

15 CONTINUE

9010

CALL BEKMTXIIJ)

560

570

580

10 CONT INUE

590

$T M=J J * D E L T$

600

610

CALL SOLCON I IWRITI

640

$\int T=J J$

IF 1 JJ .NE (JTMAX + 1) AND. ITDEP. NE - 0 ) GO TO 15

IF IJJPLE. JTMAX I GO TO 10

20 CONT INUE

IFINPROB. GT . 1) GO TO 1

690

700

710

720

500 FIRMAT $(915)$

730

513 FORMAT $(1 H O, 30 \mathrm{X}, 1$ TIME $=1$, E $12.5,1 / 1$

- 14 FIRMAT (6) I6.E14.6))

STOP

END 
-H IFTIONS - NAFE = MAIN, OPT $=02$, I. INECNT $=60$, SI ZE $=0 O O O K$,

SOUFCE, EBCDIC, NOLIST, NODECK, LOAD, MAP, NOEUIT, NOID, NUXREF

SURROUT INF INPUT

IMPLICIT REAL $+8(A-H, O-Z)$, INTEGER II-N)

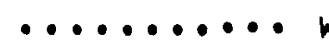

...............

$\ldots \ldots \ldots \ldots$

$\bullet \cdot \bullet \cdot \bullet \cdot \bullet \cdot \bullet$

................

...............

(..........

WHEN ELEMENT

CONNECTION

WHEN NODAL POINT CARDS ARE OMITTED,

WHEN NODALOPOINT CARDS ARE OMITTED

NEXT SPECIFIED POINT. FOR GENERATED POINTS, FXTERNAL .... HEAT FLOW IS SET EQUAL TO ZERO०-

ELEFENT ARRAY

COMMON BEQMTX(240,120),HEAT(1500), TOL, MBAND,MIT

COMMON/TVES, CORDS $(1500,2)$, NELCON(310,8), IEIMAT(310)

COMMON/TVEY NELEM, NELMAT, NNODES

COMMON/PROP, COND(4,2,2),CP(4),DEN(4), GHGEN(4)

1 , SLOPE $14,2,2), \$ L O P 2(4,2,2), A N G(310)$

COMMON/TBOUN! CFC(50), TCONV $(50)$, TCONS(1500), NCB, NCBN(50,4),

1 ITYPN $(1500)$

COMMON/DELCOM/ OELT,TM,JT, JTMAX, IPR

COMMON /VARI' IAMT, JTROL, JCNT, ITDEP

DIMENSION NAME $(18)$

$\mathrm{c}^{-}$

READ 500, NAME

PRINT 510, NAME

c.

RFAD 5?0, NNODES, NELEM, NCB, NELMAT, IDENT, TPRN

$\ddot{c}$

PRINT

$58 \mathrm{C}$, NNODES, NELEM, NCB, NELMAT

$\mathrm{C}$

READ MATERIAL PROPERTIES

WFITE $(6,550)$

RE $\triangle D(5,5404(L,((C O N D)(N, I, J), I=1,2), J=1,2), C P(N), D E N(N), G H G E N(N)$,

1 N=1, NELMAT)

WRITE $(6,551)$ (N, ( $\overline{E O N D}(N, I, J), I=1,2), J=1, ?), C P(N), O F N(N)$,

\$GHGEN (N),N=1, NELMATT

C

IF (ITDEP .NE I) GÖTÖ 7004

RFAD $7000,(1(\operatorname{SLOPE}(\mathrm{N}, I, J), I=1,2), J=1,2) ; \mathrm{N}=1, \mathrm{NELMAT})$

7000 FORMAT (4E20.4)

READ 7000, (1(SLOPZ(N,I,J), I=1, 2), J=1, 21, N = 1, NELMAT)

PRINT 7002

PRINT 7ON,; $(\mathrm{N},($ (SLOPE $(N, I, J), I=1,2), J=1,21, \mathrm{~N}=1, \mathrm{NELMAT})$

7OG2 FORMAT 1'O', $2 X$, 'MAT'; 10X. 'INITIAL CONDUCTIVITYI,5X, 'CHANGF W.R

1.T. TEMPERATURE'.)

PRINT 7003

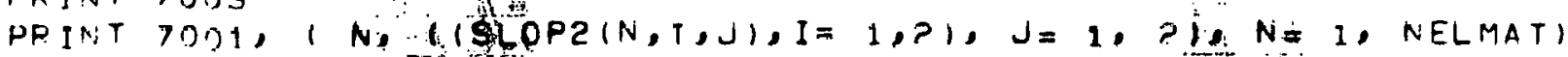

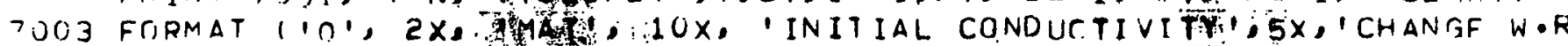
1.T. TEMPERATURE TEMAERATURE',
760

770

780

790

800

810

820

830

840

850

860

870

880

890

900

910

920

930

1020

1050

1080

1090

1100

1140

1150

1160

1170

1180

11

1190

1200

1210

1220

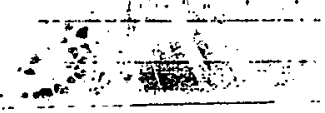

". 
$\because$ I FCDMAT ! 1:1, 1S, 4F15.4)

$7: 4$ IF I MIT EL . U, GO TO 200

1240

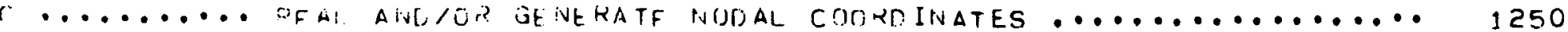

TC $\quad \mathrm{GCC}=0$

1260

$K C=1$

to RFAD SRU, N,JIG,ITYPIN(N), ICORUS (N,J),J=1,2), TCONS (N)

$I G C=I S C+1$

IFIIGC PLF. N) GO TO 61

1280

DEJNT 65O, IGC

650 FORMATI1H, $/ 1$ DATA OUT OF DRDER AT NODE1, I5,/)

$\operatorname{TTAX}=1$

1330

1340

$61 D N D D=N+1-K C$

IF $(N-K C) \quad 20,80,70$

1350

1360

c

$70 M C=K C-1$

$D X=(C O R D S(N, 1)-C O R O S(M C, 1)) / D N O D$

1370

1380

$O Y=(C O R D S(N, 2)-C O R D S(M C, 2)) / O N O D$

1390

C

$75 I T Y P N(K C)=\theta$

$I F(J I G \cdot G T \cdot 0) I T Y P N(K C)=I T Y P N(M C)$

1400

1410

$I G C=I G C+1$

$M C=K C-1$

CORDS $(K C, 1)=$ CORDS $(M C, 1)+D X$

CORDS $(K C, 2)=$ CORDS $(M C, 2)+O Y$

1420

1430

1440

1450

1460

$T C O N S(K C)=0.0$

IF (JIG AGT O) TCONS $(K C)=T C O N S(M C)$

1470

1480

1490

C

$80 K C=K C+1$

IF $(N-K C) \quad 90,80,75$

90 IF (NNODES+1-KC) $100,100,60$

1500

1510

1520

190 CONTINUE

C

$\mathrm{C}$

C

LEN $=0$

1530

1540

1550

1560

1570

1580

I $H H=0$

1590

$N E L=0$

DO $175 \mathrm{~N}=1$, NELEM

I HHEIHH+I

IF (NEL $=N) 120,170,150$

120 READ 5?8, NEL, $(N E L C O N(N, J), J=1,8)$, IELMAT(N), ANG (N)

IS I $=8$

IF (NELCON $(N, 7) \cdot E Q \cdot 0)$ ISID $=6$

IF (IHH •LE. NEL) GO TO 119

1600

1610

1630

1640

30

PRINT 660, IHH

B60 FORMATI1H, /, DATA OUT OF GRCER AT ELEMENTI,15,1)

IHH $=$ NEL

PRINT 520, NEL, (NELCON(N,J),JE1,8), IELMAT $(N), A N G(N)$

ITMAX $=1$

C

119 IF (NEL-1-N) $170,130,130$

130 IF (LEN PEO NEL) GO TO 150

Un $1.35 \quad I=1$, ISID

135 INFLCON (NEL, I) = NELCON(N,I)

IFLMAT $\left(N N_{-} L\right)=$ IELMAT $(N)$

$\triangle A B(N E L)=A N C$ (N)

L.FN=NEL

1660

1680

1690

40

1710

1720

1730

1740

1760

1770

1780 
$r$

C

$1 \div$ I) $M=N-1$

IF (NELCON(M, 7) -GT • O) GO TO 159

ISID $=6$

15y OO $160 \quad I=1 ; 1 S I D$

NELCON $(N, T)=N E L C O N(M, I)+2$

IF (ISID.EQ*6) GO TO 160

IF (I.EQ.4 tOR. I.EQ.8) NELCON(N,I) =NELCON $(N, I)-1$

160 CONTINUE

$\triangle N G(N)=A N G Q M)$

IFLMAT $(N)=$ IELMAT $(M)$

IF (NELEM-N-1) $180,180,170$

170 IF (IELMAT $(N)$ - LE 0 ) IELMAT $(N)=I E L M A T(N=1)$

175 CONTINUE

C

$1 \& 0$ CONTINUE

\section{....... RFal conveCTIVE BOUndary data}

IF (NCB) $200 ; 200,190$

190 DO $192 \mathrm{~N}=1, \mathrm{~N} \cdot \mathrm{CB}$

READ $580,(N C B N(N, K), K=1,4), C F C(N), T C D N V(N)$

IF( $N$ EQ. 1$)$ GO TO 192

IF (CFC(N) LE. 0.0$) \quad C F C(N)=C F C(N-1)$

IF( TCONV(N) LE. 0.0$) \operatorname{TCONV}(N)=\operatorname{TCONV}(N=1)$

C

192 CONTINUE

$\mathrm{C}$

$\mathrm{C}$

C

200 IF IIPRN - NE. 1) GO TO 220

PRINT $\quad 590$

PRINT $600,(I, I T Y P N(I),(C O R D S(I, J), J=1,2), T C O N S(I), I=1, N N O D E S)$

PRINT 610

PRINT 620, (I, (NELCON(I,J),J=1,8),IELMAT(I), ANG I I, I = I, NELFM)

IF (NCB) $280,220,210^{\circ}$

210 PRINT 630

PRINT 640, ( NCBN(I,J),J=1,3),CFC(I),TCONV(I),I=1,NCB)

C

$\mathrm{C}$

C

$5 O O$ FORMAT (18AS)

51) FORMAT (1H1525X:18A4)

5CO FORMAT (1015,F10.3)

528 FGRMAT (10T5\%F10.0)

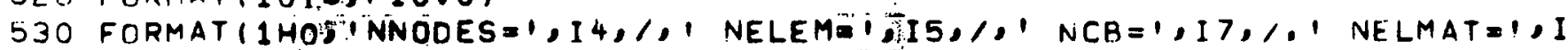
$\$ 4, /$,

540 FORMAT (15, 910.2$)$

SSC FORMATIIH $2 X, 1 M A T,, 30 X$, 'CONDUCT', 28X, ISP HEAT', 8X, 'DENSITY', 6X, SHEAT GEN:S/T).

551 FORMAT (1H,T5,7E15.4)

560 FORMATI I55.12, I3.3F10.21

$58($ FORMAT $(415.2 E 15.6)$

590 FORMATI/1,3X, 'NODES',5X, 'ITYPN', 11 X\% TROORDINATES', SGX. TTEMPERATURE,$/ 1$

SLO FRRMAT(I6,110,6X,2F10.3,E15.4)

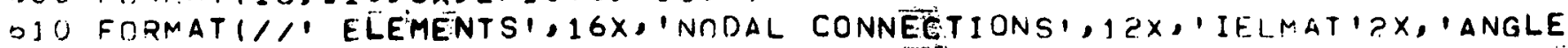

1790

1800

1810

1820

1830

1840

\section{0}

1860

1870

1880

1890

1900

1910

1920

1930

1940

1950

1960

1980

1990

2000

2010

2020

2030

2040

2050

2.060

20

80

$=110$

2140

2150

2160

60

70

2210

2230

2240

2250

2260

2270

2280

2290

2 300

2 310

2320 
(RSI-0049)

$A-7$

$1,1 / 1$

620 FORMAT $(15,9 \times, 9$ I $5, F 10 \cdot 3)$

630 FORMAT $1 / 1,4 X, 1$ NODES', $10 X, 1 F I L M$ COEFF', $2 X, 1 \triangle M B I E N T$ TEMP,,$/ / 1$ 640 FORMAT (3I5) $2 E 15.4$ )

C

220 CONTINUE

RETURN

EÑD

$$
\begin{gathered}
2330 \\
90 \\
2350 \\
2360 \\
2370 \\
2380 \\
2390 \\
2400
\end{gathered}
$$


MPILER OPTIONS - NAME $=$ MAIN, OPT $=02, L I N E C N T=60$, SI ZE $=$ OOOOK, SOUFCE, EBCDIC, NCLIST, NODECK, LOAD, MAP, NOEDIT, NOID, NOXREF

SUBROUTINF BEKMTX I JE I

$\checkmark$

c.

C

$\mathrm{C}$

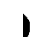

$c$

IMPLICIT REAL*8(A-H,O-Z),INTEGER (I-N)

COMMON/TVE, NELEM, NELMAT, NNODES

COMMON /TEMFS/ TDUM(1500)

COMMON/TVES, COROS $(1500,2)$, NELCON(310,8), IEI MAT(310)

COMMON/MXARG/ ORES, GP, ELCUN $(8,8), D(6,6)$

COMMON/DELCOM/ DELT,TM,JT, JTMAX, IPR

CUMMON / TBOUN: / CFC(50), TCONV (50), TCONS $(1500)$, NCB, NCBN $(50,4)$,

1 I TYPN (1500

COMMON/SOLT, HKD(1500),HKB 19500$)$

COMMON/PROP, COND(4,2,2),CP(4), DEN(4), GHGEN(4)

1 , SLOPE 14,2 , 21) - SLOP2 $(4,2,2), A N G(310)$

COMMON BEQMTX(240,120), HEAT(1500), TOL, MBAND, MIT

COMMON/HAL, $\triangle(8), Q G(8)$

COMMON /BLK, NUMBLK, NB, NMM, NL, NH, KSHIFT

COMMON /VAR1/ IAMT, JTROL, JCNT, ITDEP

DIMENSION XCOND $(310,2,2), T(2,2)$, SAVE $(2,2), L C(8)$

RFWIND 10

MAXBAN $=120$

$M A X D I F=0$

DO $5 I=1$, NELEM

$L T M=8$

IF (NELCON (I, 7) •EQ.0) LIM=6

DO $5 \mathrm{~J}=1, L \mathrm{IM}$

DO $5 K=1, L I M$

$L L=I A B S(N E L C O N(I, J)-N E L C O N(I, K))$

IF (LL, GT. MAXDIF) MAXDIF $=L L$

5 CONTINUE

MBAND $=$ MAXNIF+1

IF IMBAND LLE. MAXBAN, GO TO 7

PRINT 7373; MBAND

7373 FORMAT 1 , 11 , BANOWIDTH $=1$, I5, I TOOOO LARGE1,//1

STOP

7 CONTINUE

$c$

IF (ITDEP.FQ.1) GO TO 22

DO $11=1$, NELMAT

DO $1 J=1$, ?

DO $1 K=1$, ?

$\operatorname{SLOPE}(1, J, K)=0.0$

$1 S L O P 2(I, J, K)=0.0$

C

¿2 DO $700 \quad I=1$, NELEM

$C S=D C O S(A N G(I) * 0.01745329)$

$S S=D S I N(A N C$ II $) * 0.01745329)$

C

$T(1,1)=C S$

$T(2,1)=S S$

$T(1,2)=-S S$

$T(2,2)=C S$

NN $=$ IELMATII)
2410

$24 ? 0$

2430

2440

2450

2460

2490

2570

2580

2.620

2630

2650

2660

2670

2680

2690

2700

2710

2720

2730

2740

2750

2760

2770

2790

270

120

130

140

150

160

170

180

19 ก

200

$21 \mathrm{C}$

220

$23 \mathrm{C}$

240

250

? 60

?81r: 
TOLP $=0 . n$

$k K=0$

DO $750 \mathrm{~J}=1$, 6

IF ( NELCON(I,J) EEQ. O , GO TO 750

$K K=K K+I$

IF (MIT.GE.O ANU. JT・EQ.1, TUUM(NELCON(I,J)) $\Rightarrow$ TCONS (NELCON(I,J))

TOLD = TOLO + TOUM(NELCONII,J)

750 CONTINUE

TOLD $=$ TOLO, DFLOAT $(K K)$

$00775 \mathrm{~J}=1,2$

DO $775 K=1,2$

775 SAVE $(J, K)$

$=\operatorname{SLOP}(N M, J, K) * T O L D * 2+\operatorname{SLOPF}(N M, J, K) * T O L D+$ COND

$1 \quad(N M, J, K)$

DO $781 \quad J=1,2$

DO $781 \quad M=132$

XCONDiI, J,Mi $=0,0$

DO $781 K=132$

$D O 781 L=132$

$781 \times C O N O(I, J, M)=X C O N D(I, J, M)+T(J, K) * S A V E(K, L) * T(M, L)$

C

700 CONTINUE

8 DO. $10 \quad I=1$, NNODES

$H K D(I)=0.0$

$H K B(I)=0.0$

10 CONTINUE

LIMIT $=2 *$ MBAND

DO $14 I=$ I $;$ LIMIT

DO $14 \mathrm{~J}=\mathrm{I}$; MBAND

14 BEQMTX(I,J)=0.0

DO 1 i $I=1,6$

DO $11 \mathrm{~J}=1,6$

$D(I, J)=1 \cdot / 12 \cdot$

IF (I EQ.J) $0(1, J)=2, / 12$.

C

11 CONTINUE

DO $12 I=1,3$

DO $12 \quad J=4,6$

$D(J, I)=0.0$

$12 D(I, J)=0 . n$

ND = MBAND

NUMBLK $=0$

25 NUMBLK $=$ NUMBLK + 1

NMM = ND * NUMBLK

$N L=N M M-N D+1$

C

KSHIFT = NE -1

C

C

$\mathrm{C}$

........ CONTRUCT CONDUCTIVITY MATRIX FOR EACH ELEMENT FROM .....

2830

2840

2850

2860

2880

2890

2910

280

300

310

320

340

350

2950

2960

2970

2990

3000

3010

3020

3030

3050

070

3080

100

3110

120

130

1170

180

190

210

3220

3240

3250

3260

3270

OO $180 N=1$ SNELEM

3280

3290

3300

$M N U M=I E L M A T\{N\}$

3310

DO $785 I=1,2$

3320

$D O 785 \mathrm{~J}=1,2$

3330

SAVE $(I, J)=$ CONO $(M N U M, I, J)$

785 CONO (MNUM,I,J) $-X \operatorname{COND}(N, I, J)$

3350 
IF $(N E L C O N(N, 7) \cdot E Q \cdot 0) L I M=6$

DC $311=1$, LIM

IF (NELCON(N,I) - NL) 31, 30, 30

30 IF ( NELCON+N,I) - NMM) 32, 32, 31

31 CONTINUE

GO TO 181

32 CONTINUE

IF ILIM.EQ.8) GO TO 73

CALL CONMTMIN)

GO TO 74

73 CALL INTGIN,

C

C

Go TO 20

$740049 I=2, t I M, 2$ :

$N 1=N E L C O N(N ; I)$

IF (INI.LTENL) OOR (N1.GT.NMM)) GO TO 49

50 HKO(NI) $=$ HKDINI) \& QRES

$H K B(N 1)=H K B(N 1)+O P$

C

49 CONTINUE

$60 \quad$ TO 28

$200021 \quad I=1, K I M$

NI =NELCON $(N, I)$

IF (INI・LT•NL) OOR: (NI:GT・NMM)) GO TO 21 HKD (NI)=HKD (N1) DQ

AKB $(N I)=H K O ̈(N I)+A T I)$

C...? 2 CONTINUE

C

60 T0 69

C.

28 DO $2 \quad K=1,3$

$I J=2 \neq \bar{K}-1$

$L C(k)=I J$

C

$2 L C(k+3)=I J+1$

C

Gö Ṫ 68

$69^{\cdots}$ Do $3 K-1,8$

$3 L C(K)=K$

C
$\mathbf{c}$
c
$\dot{C}$

ASSEMBLE OLOBAL BTIFFNÉSS

68 DO $176 \quad I=13 L I M$

II $=L C(I)$

ISAV =NELCON(N,I.I)

ISAY = ISAV - KSHIFT

IF I ISAV ILE. O .ORE IEAV .OT. NOI GOO TO 176

157 DO $175 \mathrm{~J}=1$ SEIM

$J J=L C I J T$

JJSA $=N E L C O N(N, J J)$

$J S A V=J J S A-Z S A V+1$

JSAV a JSAV - KSHIFT

165 IF (JSAV) $195,175,170$

170 BEQMTX(ISAV; JSAV) $=B E$ BMTX(ISAV, JSAV) + ELCON(I,J)
3366

337(

3386

3396

3406

3426

3436

$344 C$

$348 \mathrm{C}$

3490

$353 \mathrm{C}$

$354 \mathrm{C}$

$355 \mathrm{C}$

$356 C$

3576

$358 \mathrm{C}$

3596

$360 \mathrm{C}$

$361 \mathrm{C}$

$362 C$

$363 \mathrm{C}$

$364 \mathrm{C}$

$365 \mathrm{C}$

$366 \mathrm{C}$

$367 \mathrm{C}$

$368 \mathrm{C}$

$369 C$

$370 \mathrm{C}$

$371 \mathrm{C}$

$372 \mathrm{C}$

$373 \mathrm{C}$

$374 \mathrm{C}$

$375 \mathrm{C}$

$376 \mathrm{C}$

$377 \mathrm{C}$

$378 \mathrm{C}$

$379 \mathrm{C}$

$380 \mathrm{C}$

$381 \mathrm{C}$

3820

3830

3840

3850

3860

3870

3880

3890

3900

3910

3920

3930

3940 3950 3960 3970 3980 3990 
C

$1 / S$ CONTINUNE

176 CINTINUE.

1 : 1 CONTINIJF.

OCi $795 I=1,2$

DCj $795 \mathrm{~J}=1.2$

4001

4011

4021

370

3451

795 COND (MNUM, $1, J)=\operatorname{SAVE}(I, J)$

3461

$1 \& O$ CONTINUE

$347 i$

CALL BYCON I JE ।

4036

DO $182 \quad N=1$;MBANO

aisi

182 WRITE(10) (FEQMTX(N,M),M=1, MBAND)

DO $1901=1$, ND

DO $190 \mathrm{~J}=1, \mathrm{ND}$

190 BF.QMTX(I.J) $=0.0$

IF ( NMM ILT. NNODES , GO TO 25

RETURN

END

4061 4078

4086

$40 \%$

$410 \%$

$211 \hat{~}$

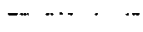

$-$
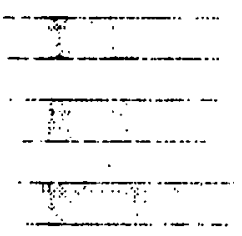

$\div$
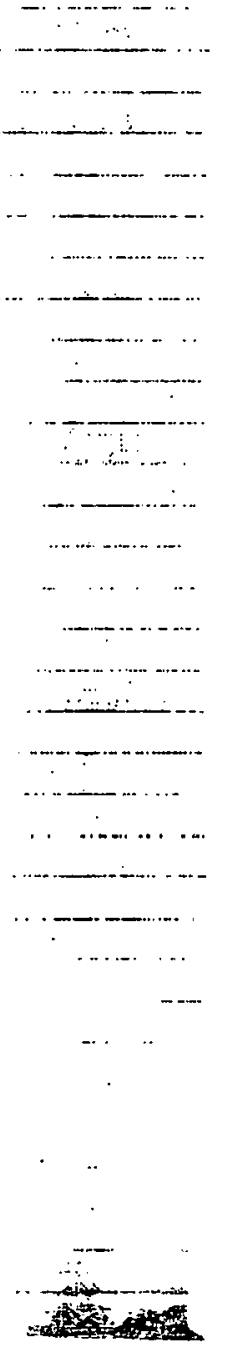
HILER UPTIONS - NANE = MAIN, OPT $=02$, LINECNT $=60$, SIZE $=0000 \mathrm{~K}$,

SOUFCE, EBCUIC, NOLIST, NODECK, LOAD, MAP, NOEOIT, NOID, NOXREF

SIJBRDUTINF FEYCON I JE I

6410 6420

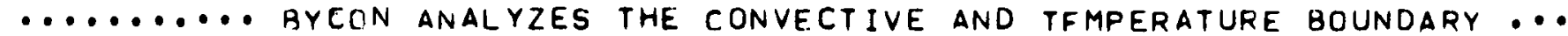

$\ldots \ldots \ldots \ldots$ CONOITIONS AND INCLUDES THEM IN THE CONDUCTIVTTY $\ldots \ldots \ldots$

C $\ldots \ldots \ldots \ldots . .$. MATFIX FOR THE WHOLE BODY (BEQMTX) $\ldots \ldots \ldots \ldots \ldots \ldots \ldots \ldots \ldots$

C

C

IMPLICIT REAL*8(A-H,O-Z), INTEGER I I-N)

COMMON/TVEA NELEM, NELMAT, NNODES

COMMON/TVES, CORDS $(1500,2)$, NELCON $(310,8)$, IEI.MAT $(310)$

COMMON/PROP, COND $(4,2,2), C P(4), D E N(4)$, GHGEN(4)

1 , SLOPE $(4) 2,2), \operatorname{SLOP} 2(4,2,2)$

COMMON/TBOUN/ CFX(50), TCONX(50), TCONS(1500), NCB, NCBN(50,4),

1 ITYPN (1500)

COMMON/SOLT, HKD(1500),HKB(1500)

COMMON/MXARG/ QRES,OP, ELCON $(8,8), D(6,6)$

COMMON BEQMTX(240,120),HEAT(1500), TOL, MBAND, MIT

COMMON /BLK/ NUMBLK, NB, NM, NL, NH, KSHIFT

DIMENSION 18$), 8(2,8), Z N(8), Z N X I(8), Z N E T(8)$

DIMENSION CFC $(50)$, TCONV $(50)$

C

C

IF (JE) $70,70,10$

C

C

$\mathrm{C}$

$10 \triangle A 1=-0.5973502692$

$A G 2=0.5973502692$

$A G 3=-1 \cdot 0$

$A G_{4}=1 \cdot 0$

DO $69 \mathrm{~N}=\&, \mathrm{NCB}$

DO $800 I=1,3$

IF ( NCBN(N,I)-NL) 800,801,801

801 IF ( NCBN(N,I)-NM) $802,802,800$

800 CONTINUE

GO TO 69

C

802 CONTINUE

$N O D_{1}=N C B N(N, 1)$

$N O D 2=N C B N(N, 2)$

NOD3 $=N C B N+N, 31$

$\mathrm{NOD} 4=\mathrm{NCBN}+\mathrm{N}, 41$

NODX = NODI-KSHIFT

NODY = NODE - KSHIFT

NOOZ $=$ NODB - KSHIFT

IF (NELCON $(N O D 4,7)$.NE.O) GO TO 68

HYP $=$ DSQR T ( (CORDS $($ NOD 3,1$)=$ CORDS $(N O D 1,1)) * * 2+(\operatorname{CORDS}($ NOD 3,2$)-\operatorname{CORDS}($

1 NOO1, $11 * * 21$

C

$30 \operatorname{TCDNV}(N)=C F \times(N) * H Y P * T C O N \times(N) / 3$

$C F C(N)=C F \times(N) * H Y P / 30$.

C

IF $((N O D X \cdot L E \cdot O) \cdot O R \cdot(N O D X \cdot G T \cdot M B A N D))$ GO TO 31

$H K B(N O D)=H K B(N O D 1)+T C O N V(N) / 2$.

31 IF ( (NODY.EE・O) .OR (NOUY・GT.MBAND)) GO TO 3 ?

$H K A(N O D Z)=H K B(N O D 2)+T C O N V(N) * 2$.
6430

6440

6450

6460

6470

6480

6540

6560

6570

6590

6600

6610

6620

6630

6640

6660

6670

6680

6690

6700

6710

6720

6730

6740

6750

6760

6770

6780

6790

6800

6830

6860

6870

6880

6890

6900 


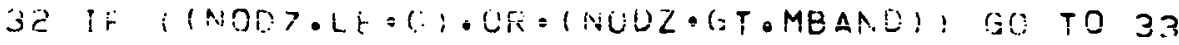

HK. $(N O D 3)=H K H(N O D 3)+T C O N V(N) / \ddot{Z}$.

33 CONTINUE

IF ( (NODX $: L E \cdot O) \cdot U R \cdot(N O D X \cdot G T \cdot M B A N D))$ GO TO 150 BE OMTX(NODX, 1$)=B E Q M T X(N O U X, 1)+C F C(N) * 4 \cdot 0$

6940

150 IF (INODY ILE. O) .OR-(NODY.GT.MBAND)) GO TO 160 BE DMTX(NODY, 1$)=B E(N M T X(N O D Y, 1)+C F C(N) * 16.0$

6960

160 IF ( (NODZ LE. O ) OR (NODZ GT.MBAND)) GO TO 170 BEQMTX $(N O D Z, 1)=B E Q M T X(N O D Z, 1)+C F C(N) * 4 \cdot$ C

170 CONTINUE

6980

6990

7000

$L L=N O D Y-N \cap O X+1$

IF (LL) $50,50,40$

7010

7020

7030

7040

GO TO 60

$50 L L=N O D X-N O D Y+1$

BE.QMTX $(N O D Y, L L)=B E Q M T X(N O D Y, L L)+C F C(N) * 2 \cdot 0$

60 CONTINUE

$L L=N O D Z=N O D X+1$

IF (LL) 61; 61, 62

62 BEQMTX (NODX, LL) = BEQMTX NODX,LL) - CFC(N)

GO TO 65

$61 \mathrm{LL}=\mathrm{NODX}-\mathrm{NODZ}+1$

BEOMTX(NODZ, LL) = BEQMTX(NODZ, LL) - CFC(N)

$65 \mathrm{LL}=$ NODY $-\mathrm{NODZ}+1$

IF (LL) $63,63,64$

64 BEOMTX(NODZ; LL) = BEQMTX(NODZOLL) + 2. * CFC(N)

GO TO 69

7050

7060

7070

7080

7090

7100

7110

7120

7130

7140

7150

7160

7170

$03 \mathrm{LL}=\mathrm{NODZ}$ - NODY + 1

BEQMTX(NDDF; LL) = BEQMTX(NODY, LL) + 2**CFC(N)

7180

GO TO 69

7190

7200

7210

CONTINUE

7220

$A(I I)=0.0$

7230

DO 1 J J $=1,8$

7240

7250

$1 \quad$ ELCONIII,JUI $=0.0$

7260

DO $900 \mathrm{~J}=132$

IF (NODZ - NE. NELCON(NOD4,2)) GO TC 898

7270

JJ $=3$

I I $=\mathrm{J}$

GO'TO 895

7280

7290

7300

7310

7320

7330

II $=4$

$\mathrm{JJ}=\mathrm{J}$

7340

GO TO 895

7350

7360

7370

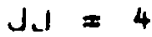

$I I=J$

7380

GO TO 895

7390

89E IF(NOD2 .NE. NELCON(NOD4,8)) GO TO 894

$I I=3$

7410

$J \mathrm{~J}=\mathrm{J}$

7420

7430

GO TO 895

X94 PHINT 1000 N NOUT

1000 FGRMAT1 $1,10 \times, 1 * * * *$ NOUZ EQUALS', I 10, 'WHICH IS IN ERROR', 
KYS CALL SHAPEAII, JJ, DETJAC,NUD4,AGI,AGZ, AG3,AG4,B,ZW, Z, ZNXI,ZNET)

SY $=0.0$

$S X=0.0$

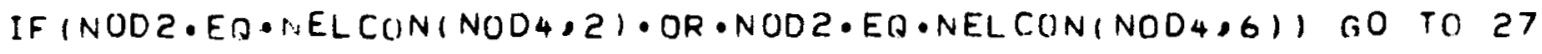

$D O 26 \quad L R=1,8$

NOD=NELCON $(N O D 4, L K)$

$S X=Z N E T(L R) * \operatorname{CORDS}(N O D, 1)+S X$

26 SY $=Z N E T(L R) * C O R D S(N O D, 2)+S Y$

GO TO 29

$27 \quad D O 28 L R=1,8$

$N O D=N E L C O N(N O D 4, L R)$

$S X=Z N X I(L R) * C O R D S(N O D, 1)+S X$

28. $S Y=Z N X I(L) * C O R D S(N O D, 2)+S Y$

$29 \quad S \times 2=S X * S X$

$S Y Z=S Y * S Y$

HYP $=$ DSART $(5 X 2+S Y Z)$

7470

7480

7490

7500

7510

7520

7530

7540

7550

7560

7570

C

DO 900 NROW $=1,8$

$N N=N$

$A(N R O W)=A(N R O W)+Z N(N R O W) * C F X(N N) * T C O N X(N N) * H Y P$

DO 900 NCOE $=$ NROW: 8

$900 E L C O N(N R \cap W, N C O L)=E L C O N(N R O W, N C O L)+Z N(N R O W) * Z N(N C O I) * C F \times(N N)$

1 * HYP

DO $901 K=2,8$

DO $901 L=1 \$ K$

$901 E L C O N(K, L)=E L C O N(L, K)$

7580

7600

7610

7620

7630

7640

7650

7660

7670

7680

7690

DO $3 \quad I=1,8$

7700

N2. = NELCON(NOD4,I)

$N 1=N 2$ - KSHIFT

IF ( N1 .LE: O . OR. NI GT. MBAND, GO TO 3

$H K B(N 2)=H K B(N 2)+A(I)$

DO $4 \mathrm{~J}=1 \% 8$

N21 = NELCON(NOD4,J)

$N 2=N 21-N 1+1-K S H I F T$

IF (N2) 4,435

5 BEOMTX(N1,NC̈) = BEOMTX(N1,N2) + ELCON(I,J)

4 CONTINUE

3 CONTINUE

2 CONTINUE

69 CONTINUE

70 CONTINUE

$c$

C (2)

$$
\begin{aligned}
& \text { LIMIT = NM + MBAND } \\
& \text { DO } 140 N=N L, L I M I T \\
& \text { IF } 1 N \text {.GT. NNODES, GO TO } 140
\end{aligned}
$$

7710

7750

7760

7770

7780

7790

7800

7810

7820

7830

7840

7850

7860

7870

7880

7890

7900

7910

7920

IF (ITYPN (N) $\angle T \cdot O)$ GO TO 140

7930

$H K B(N)=H K B(N)+T C O N S(N)$

IF(I TYPN(N) $140,140,80$

7940

7950

7960

*O DO $130 \quad M=? ; M B A N D$

$L=N-M+1$

$J=L-K \operatorname{Sin} I F T$

IF ( $(J \cdot L F \cdot O) \cdot O R \cdot(J \cdot G T \cdot M B A N D)$ GO TO 100

7970

7980

7990

8000

IF (L) $100,160.90$

8010 
(RSI-0049)

SO HKS(L) =HKPAL) - SHOMTXIM:M)*TCONS!N!

$(A-15)$

BF (JMTX(J,M) $=0 \cdot 0$

$100 \mathrm{LL} .=N+M-1$

$J=N \rightarrow K S H I F T$

IF $((J \cdot L E \cdot G) \cdot U K \cdot(J \cdot G T \cdot M B A N D))$ GO TO 130

IF (NNODES-EL) $120,110,110$

$110 H K B(L L)=H K B(L L)-B E O M T X(J, M) * \operatorname{TCONS}(N)$

$1 \approx 0$ BFQMTX $(J, M)=0 \cdot 0$

130 CONT INUE

$J=N-K S H I F T$

IF ( $(\mathrm{J} \cdot L E \cdot O), O R \cdot(\mathrm{J}, \mathrm{GT} \cdot \mathrm{MBAND})$ (GO TO 140

C

BF $\triangle M T \times(J, 1)=1 \cdot 0$

HKB $(N)=T C O N S(N)$

140 CONT INUE

RE TURN

END

8020

8030

8040

8050

8060

8070

8080

8090

8100

8110

8120

8130

8140

8150

8160

8170

8180 
'ILEK OFTIONS - NAME $=$ MAIN,OPT $=02$, LINECNT $=60$, SIZE $=0000 \mathrm{~K}$, SOURCE, EBCDIC, NOLIST, NODECK, LOAD, MAP, NOEOIT, NOID, N IJXREF

SUBROUTINE CONMTX(N)

4120

4130

4140 4150

IMPLICIT REAL*8(A-H,O-Z), INTEGER $(I-N)$

COMMON/TVEO NELEM, NELMAT, NNODES

COMMON/TVEG, CORDS $(1500,2)$, NELCON $(310,8)$, IEI MAT(310)

COMMON/PROP, COND $(4,2,2), C P(4), D E N(4), G H G E N(4)$

1 . SLOPE $(452,2), \operatorname{SLOP} 2(4,2,2)$

COMMON/TBOUN/ CFC(50), TCONV(50), TCONS $(1500)$, NCB, NCBN(50,4),

1 ITYPN 1200 .

COMMON/SOL T, HKD(1500),HKB(1500)

CONMON/OELEOM/ DELT, TM,JT, JTMAX, IPR

COMMON/MXARG/ QRES, QP, ELCON $(8,8), 0(6,6)$

COMMON BEOHTX(240,120),OHEAT(1500), TOL, MBAND,MIT

REAL $\$ 8$ KMAT $+6,61$

DIMENSION DN $(6,6), X L C O N(6,6)$

C

C

DO $10 \quad I=1,6$

DO $10 \mathrm{~J}=1,6$

KMAT $(I, J)=0.0$

$10 \operatorname{ELCON}(I, J)=0.0$

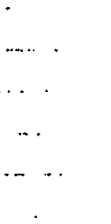

C

NODDI $=$ NELCON $(N, I)$

NODS $=$ NELCON+N,3)

NODS $=N E L C O N\{N, 5$ )

NODT =NELCON\&N,7)

MNUMEIELMAT(N)

C

DO $11 \quad 1=1,6$

CONI =COND $(M N U M, 1,1)$

- IF(I.GT.3) CONI=COND(MNUM.2,2)

$c$

$11 \operatorname{KMAT}(I, I)=C O N I$

C

KMAT $(1,4)=$ COND(MNUM, 1,21$)$

KMAT $(\overline{2}, 5)$ - COND(MNUM,1,2)

KMAT $(3,6)=$ COND (MNUM, 1,2$)$

KMAT $(4,1)=$ COND(MNUM, 2, 15

KMAT $(5,2)=$ COND (MNUM, 2,1)

KMAT $(6,3)=$ COND(MNUM, 2, 1$)$

C

$12 A_{1}=$ CORDS $(N O D 5,1)-$ CORDS $(N O N O D, 1)$

$A 2=$ CORDS $(N O D 1,1)$ - CORDS $(N O D 5,1)$

$A 3=$ CORDS (NOD3,1) - COROS $(N O D 1,1)$

B1 CORDS INOD 3,2$)$ - CORDS $(N O D 5,2)$

B2 - CORDS $1 N O D 5,2)$ - CORDS(NOD1,2)

$c$

B3 = CORDS INOD1,2) - CORDS (NOD3,2)

$x=1.0$

$40 A R=A 3 * B 2-A 2 * B 3$

IFIAR $\cdot G T \cdot(\cdot 0)$ GO TO 33

PRINT $500, \mathrm{~N}$

I QUI T $=1$ 


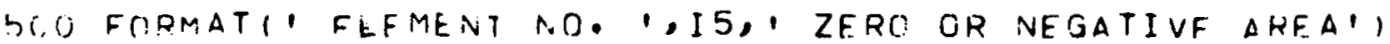

$\because 3$ QATID $=x /(P \bullet * A F)$

IF IN E EQ NELEM - AND. IQUIT EEQ 1 ) CALL EXTT $D P=X * A R * G H G E N\left(M N U N_{i}\right) / 6$.

42 QRES $=\triangle R * X * C F(M N U M) * D E N(M N U M) / 6 \cdot 0$

$D N(1.5)=0.0$

$\operatorname{DN}(2.6)=0.0$

$D N(3.4)=0.0$

$D N(4.5)=0.0$

$D N(5.6)=0.0$

$D N(6.4)=0.0$

C

$O N(1,1)=3 \cdot * E 1$

$D N(1.2)=-82$

$\operatorname{DN}(3,2)=-62$

$D N(1,3)=-B 3$

$D N(2,3)=-63$

$D N(1.4)=4 . * B 2$

$D N(3,5)=4 . * B 2$

$D N(1.6)=4 . * 83$

C

$D N(2,5)=0 * 43$

c

$D N(2,1)=-B 1$

$D N(3,1)=-B 1$

$D N(2,2)=3 . * 82$

ON $(2.4)=4 . * B 1$

$\operatorname{DN}(3.6)=4 . * 81$

4780

$D N(3,3)=3 \cdot * 83$

4820

4830

4840

$D N(4,1)=3 . * A 1$

$D N(4,2)=-A 2$

$D N(6,2)=-A 2$

$D N(4,3)=-A 3$

$D N(5,3)=-A 3$

$O N(4,4)=4 . * A 2$

UN $(6,5)=. .425$

$D N(4,6)=* * 43$

$D N(5,5)=* * A 3$

C

$D N(5,1)=-A 1$

DN $(6,1)=-A 1$

$D N(5,2)=3 . * A 2$

$D N(5,4)=4 . * A 1$

4850

$D N(6,6)=0.7 A 1$

ON $(6,3)=3 \cdot A A 3$

4900

4920

FORM CONDUCTIVITY MATRIX

4940

4950

4960

4970

DO $88 \quad I=1.6$

DO $88 \mathrm{~J}=1,6$

560

570

$X L C O N(I ; J)=0 \cdot 0$

590

$88 \times I . C O N(I ; J)=\operatorname{XLCON}(I, J)+\operatorname{KMAT}(I, K) \neq D N(K, J)$

DO $90 \quad I=1.6$

DO $90 \quad J=1, I$

ELCON $(I, J)=O \cdot O$ 
(RSI-0049)

$(A-18)$

()) $50 \quad k=1, t$

5010

Ur: $30 \quad L=1,6$

5020

5030

- ELCON(I,J)=ELCON $(I, J)+\operatorname{DN}(K, I) * U(K, L) * X L C O N(L, J) * R A T T O$

5060

DO $91 I=1,6$

DC $91 \mathrm{~J}=1$; I

5070

5080

$91 E(\operatorname{CON}(J, I)=\operatorname{ELCON}(I, J)$

5090

RFTURN

END

5100

5110 
APILER OFTIONS - NAIEE = MAIN, OPT=02, LINECNT =60, SIZE= OOOOK,

SNLFCE, EBCDIC, NOL IST, NODECK, LOAD, MAP, NOFOI T, NOID, NOXREF

SLRROUTIVF. INTG(M)

IMPLICIT REAL $\# 8(A-H, O-Z)$, INTEGER $(I-N)$

REAL $* 8 \quad N, N \times I, N E T$

CCMMON/TVES, COROS $(1500,2), N E L C O N(310,8)$, IEL MAT(310)

COMMON/MXAFG/ ORES, QP, ELCON $(8,8), D(6,6)$

COMMON/HAI_, $A(8), O G(8)$

COMMON/PROF, CONO(4,2, 2i,CP(4),DEN(4), GHGEN(4)

1 , SLOPE $(4,2,2), \operatorname{SLOP} 2(4,2,2)$

DIMENSION $(2,8), N(8), C O N X(2,2), N X I(8), N E T(R)$

C

$M 1=I E L M A T(M)$

c

DO $6 K=1,8$

$B G(K)=0.0$

$A(K)=0.0$

DO $6 L=K, 8$

$6 E L C O N(K, L)=0 \cdot 0$

C

$\operatorname{CONX}(1,1)=\operatorname{DSQRT}(\operatorname{COND}(M), 1,1))$

$\operatorname{CONX}(1,2)=\operatorname{COND}(M 1,1,2) / \operatorname{CON} \times(1,1)$

$\operatorname{CON} \times(2,2)=$ DSQRT(COND $(M 1,2,2)-\operatorname{CONX}(1,2) * \operatorname{CON} \times(1,2))$

C

GAUSS DUAD. LOOP

C

AREA $=0.0$

$A G 1=-0.5973502692$

AG2 $=0.5973502692$

$\triangle G 3=0.0$

DO $50 \quad I I=13.2$

DO $20 \quad J J=1,2$

CALL SHAPEIII, JJ,DETJAC, M, AG 1, AG2, AG 3, AG 3, B, N, O,NXI, NFTI

IF 1 DETJAC ILE. 0.001 PRINT 1000, M, DETJAC

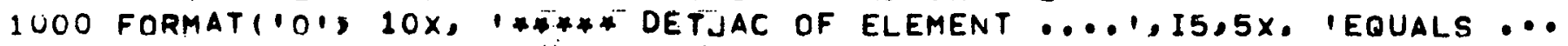
$11, E 15 \cdot 3,5 x, 1 * * * * * 11$.

C

DO $9 K=1,2$

DO $9 L=1,8$

C

DuMi $=0$.

9

DO $8 \mathrm{MM}=K, 2$

8 DUM1 $=$ DUM + CONX $\quad(K, M M I * B(M M, L)$

C

$B(K, L)=D U M 1$

DUM1 = DETUAC

$\triangle R E A=A R E A+D U M 1$

C

DO 20 NROW 1,8

$D G(N R O W)=D G(N R O W)+N(N R O W) * D E N(M 1) * C P(M 1) * D U M 1$

$\triangle(N R O W)=A+N R O W)+N(N R O W) * G H G E N(M 1) * D U M 1$

17 CONTINUE

DO $20 \mathrm{NCOL}=\mathrm{NROW}, 8$

$D U M ?=0$.

$D C 18 L L=1>2$.

18. DUM? $=D U M 2+B(L L, N R O W)+8 \cdot(L L, N C O L)$

C

¿U EI CONINROW,MCOL) = ELGON(NROW,NCOL) + DUMI *OUMP

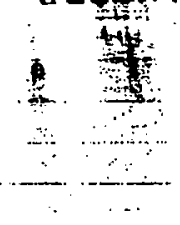


i) $30 k=2,8$

5640

): $30 L=1, K$

5650

$\therefore E(\operatorname{CON}(K, L)=E L C U N(L, K)$

5660

FURM LIIMFED HEAT CAHACITY MATRIX $11 / 4$ ON SEC. NODFSI

5670

5680

5690

JHES $=$ DEN(MI) * CF(MI) * AREA / 4.0

$\operatorname{LOG}(1)=0.0$

$D G(3)=0.0$

$J G(5)=0 . C$

$D G(7)=0.0$

QG(2) = DRES

$O G(4)=$ DRES

i)(j(6) = DRES

c

$D G(R)=D R E S$ 
MILILEK JPTTONS - NAMIF $=$ MAIN, UPT $=02$, LINECNT $=60$, SI ZE $=0000 \mathrm{~K}$,

SIUFCE, EBC UIC, NOLIST, NODECK, LOAD, MAP, NUFDIT, NOID, NOXREF

SUBROUTINF. SHAPE $(I I, J J, D E T J A C, M, A 1, A 2, A 3, \triangle 4, B, N, M O R, N X I, N E T$ )

5760

IMPLICIT REAL $\$ 8(A-H, O-Z)$, INTEGER I I-N)

COMMON/TVES, CORDS $(1500,2)$, NELCON $(310,8)$, IELMAT(310)

CCIMMON/MXARG/ QRES, QP,ELCON $(8,8), D(6,6)$

5780

REAL *8 N(R), NXI (8), NET(8),JAC(2, 2$)$

DIMENSION $\triangle A(4), B(2,8)$

5800

C

$$
\begin{aligned}
& A \Delta(1)=A 1 \\
& A A(2)=A 2 \\
& A A(3)=A 3 \\
& A A(4)=A 4
\end{aligned}
$$

5810

5820

5830

5840

5850

5860

$N(1)=0.25 *(1--A A(I I))+(1--A A(J J)) *(-A A(I I)-A A(J J)=1-1)$

5870

$N(2)=0.5 *(1 .-A A(I I) * A A(I I)) *(1-0 A A(J J))$

5880

$N(3)=0.25 *(1++A A(I I)) *(1,-A A(J J))+(A A(I I)-A A(J J)-1 \cdot)$

$N(4)=0.5 *(1 \cdot+\Delta A(I I)) *(1 \cdot-A A(J J) * A A(J J))$

$N(5)=0.35 *(1 \bullet+A A(I I))+(10+A A(J J)+(A A(I I)+\Delta A(J J)-1 \cdot)$

$N(6)=0.5 *(1 . * A A(I I) * A A(I I)) *(1 *+A A(J J))$

$N(7)=0.25 *(1,-A A(I I)+(10+\Delta A(J J I) *(-A A(I I)+A A(J J)-1 \cdot)$

$N(8)=0.5 *(1 \cdot * A A(I I))+(1 \cdot-A A(J J) * A A(J J))$

C

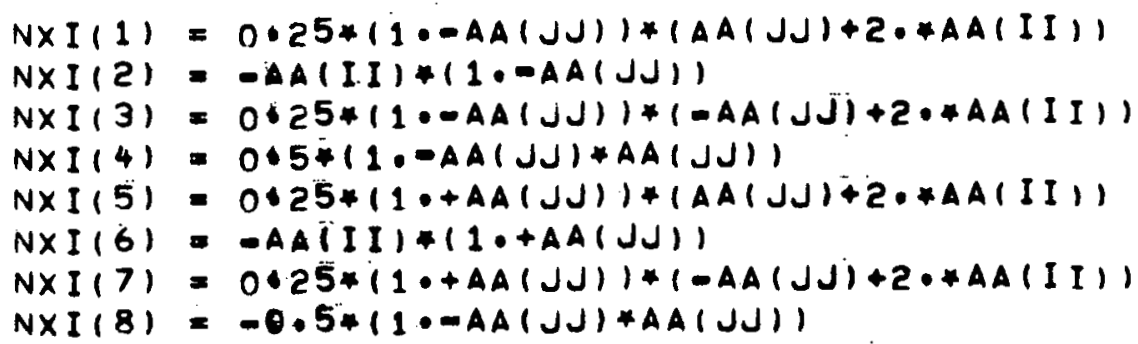

5890

5900

5910

5920

5930

5940

5950

5960

5970

5980

5990

6000

6010

6020

C

6030

NET(1) = 0.25*(1--AA(II) $*(A A(I I)+2 \cdot * A A(J J))$

NET(2) $=-0.5 *(1-0 A(I I)+A A(I I))$

NET $(3)=0 * 25 *(1 \cdot+A A(I I)) * 1-A A(I I): 2 * * A A(J J))$

NET(4)= IAJJ)+(1-+AA(II)

$N E T(5)=0+25 *(1 \cdot+A A(I I)) *(A A(I I)+\overline{2} * * A A(J J))$

NET(6)=0.5*(1.-AA(II)+AA(II)

NET $(7)=0$ ) $5 *(1--A A(I I)) * 1-A A(I I)+2 * * A A(J J)$ )

NET $(8)=-A A(J J) H I I \cdot-A A(I I))$

6040

6050

6060

6070

6080

6090

6100

6110

6120

JACOBIAN, UNVERSE JACOBIAN, AND DET JACOBEAN

6130

6140

6150

DO $10 \quad I=1,2$

DO $10 \quad J=1,2$

10

$J \Delta C(I, J)=0$

DO $15 \quad I=1,8$

$N N=$ NELCON?M, I I

$J A C(1,1)=\operatorname{JAC}(1,1)+N X I(I)+\operatorname{CORDS}(N N, 1)$

$J \Delta C(1,2)=J A C(1,2)+N X I(I) * C O R D S(N N, 2)$

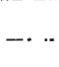

6160

6170

6180

6190

6200

6210

6220

6230

$J A C(2,1)=J A C(2,1)+N E T(I)+C O R D S(N N, I)$.

$15 J \Delta C(2,2)=J A C(2,2)+N E T(I) * C O R D S(N N, 2)$

6240

6250

DETJAC $=J A C(1,1) * J A C(2,2)-J A C(\varepsilon, 1) * J A C(1,2)$

IF $(M O R, G T \cdot 0)$ GO TO 31

DUMM $1=J A C\{1,1) / D E T J A C$

$J A C(1,1)=J A C(2,2) / D E T J A C$

$J A C(1,2)=-J A C(1,2) / O E T J A C$

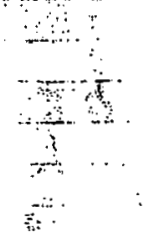

6260

6270

6280

6290 
(RSI-0049)

$A-22$

$J A C(2,1)=-J A C(2,1) / D E T J A C$

$J A C(2,2)=$ DUM1

Dก $20 \quad L=1,8$

$B(1, L)=J A C(1,1) * N X I(L)+J A C(1,2) * N E T(L)$

C

$20 B(2, L)=J A C(2,1) * N X I(L)+J A C(2,2) * N E T(L)$

31 CONTINUE

RF TURN

END

6310

6320

6330

6340

6350

6360

6370

6380

6390

6400 


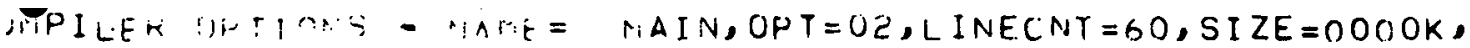

SCLICE, EBCUIC, INCLIST, NOOECK, LOAD, MAP, NOFUIT, NOIC, NOXRFF

SIFRROUTING SULCON I IWRIT ,

8200

8210

8220

8230

8240

8250

8260

8270

8280

IMPLICIT REAL*8(A-H,O-Z), INTEGER(I-N)

COMMON/TVE/ NELEM, NELMAT, NNODES

COMMON/TVES, CORDS $(1500,2)$, NELCON(310,8), IEIMAT(310)

COMMONIDELCOM, DELT, TMQUT, JTMAX, IPR

COMMON/TBOUN/ CFC(50), TCONV(50), TCONS(1500), NCB, NCBN(50,4),

1 ITYPN 115001

COMMON/SOLT/ HKD(1500),HKB(1500)

COMMON/PROP, COND $(4,2,2), C P(4), O E N(4), G H G E N(4)$

1 , SLOPE $(452,2), S L O P 2(4,2,2)$

COMMON BEDMTX $(240,120)$, HEAT(1500), TOL, MBAND,MTT

COMMON /VAK1/ IAMT, JTROL, JCNT, ITOEP

COMMON /TEMPS/ TDUM(1500)

C

c

C

IF (DELT) $100,100,10$

10 IF (JT.GT.1 IAND. ITDEP.EQ. 0$)$ GO TO 45

15 TLED $=1$ - /DEET

REWIND 1

I $\triangle M T=$ IDINTITLED I

IF (IPR - LE O OI IPR=I

IF IJT.GT.1Y GO TO 16

$J T R O L=0$

$J C N T=0$

$160040 I=4$; NNODES

IF I ITYPNIII, EE. 1, GO TO 40

HKDII $=$ HKD II) * TLED

40 CONTINUE

CALL SOLVET1, NNODES I

c

C
$45 J T R O L=J T R O K+1$

$J C N T=J C N T+1$

DO $60 J=1$, NNODES

IF (MIT•LT.Q •OR. JT.NE・1) GO TO 48

$\operatorname{TUUM}(J)=T L O N S(J)$

48 CONTINUE

HEAT $(J)=H K B(J)$

$* \quad 3 \cdot 0$

IF (ITYPN (J)) $50,50,60$

50 HEAT $(J)=3.0 * H K B(J)+H K D(J) * T D U M(J) * 4.5$

GO CONTINIJE

TEMPERATURES BY CONSTRUCTING EFFECTIVE LOAD ... D CALLING SOLVE ........................

$\bar{c}$
8390

8400

8410

8420

8440

8460

8480

8490

8500

8510

8520

8530

8540

8570

8590

8600

8610

8620

9040

9050

9060

9070

9090

9100

9110

9120

9130

9140

9150

9160 
CALL SOLVEIP,NNMDES)

SE JO $95 \mathrm{M}=1$, NNUDFS

9180

$\zeta$ TDUM (M) = (HEAT(M) - TDUM(M) II2.0

9190

9200

9210

9220

$\bar{c}$

IFITM LLE: $5.01, \overline{G O}$ TO 85

9230

IF (JTROL.ED.IAMT) GO TO 86

$\because \quad$ IF I JCNT $\cap N E$ IPR I GO TO 120

OS JTROL $=0$

9240

PRINT $\quad 500$ THE

IF I WRIT) $71,120,7.1$

71 INCR $=$ NNODES

75 PRINT 510 (N) TOUM (N) ,N=1,INCR) JCNT:=O

IF I IWRIT ONE 2) 00 TO 120

9260

IF $($ UT ONE. JTMAX) 60 THO IRO

9270

PUNCH 7761 , II, TOUM (I), I. 10 NNODES ,

$c$

Go TO 120

9440

c

STEAOY aTATE

Why

9450

C

C

100 DO 110 L=1.NNODFS

110 HEAT (L) IEHKB(L)

CALL SOLVEIIONNODESI

Hotosin

9460

9470

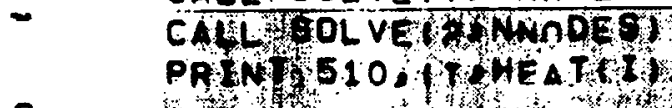

C

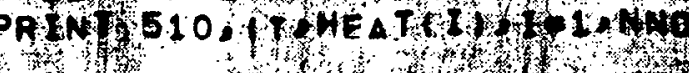

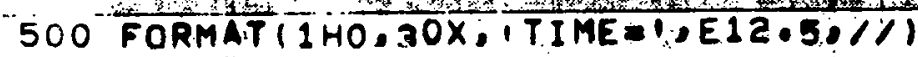

9480

9490

9500

510 FORMAT ( 8 (I .E E1?.4))

520 FORMATI//,4OX, IFLEMENTAL TEMPERATURES!,I!

$7761 \%$

C.

$220 \mathrm{Co}+\mathrm{HE}$

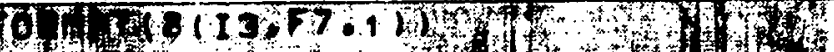

RETURiN

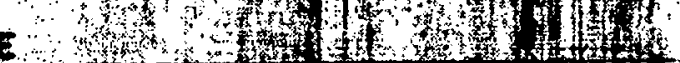

END

ख)

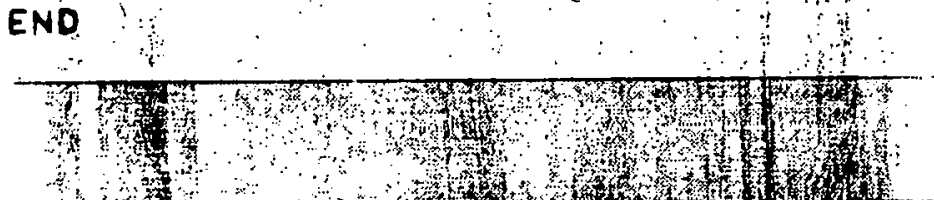

,

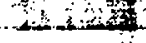

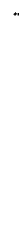

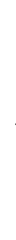

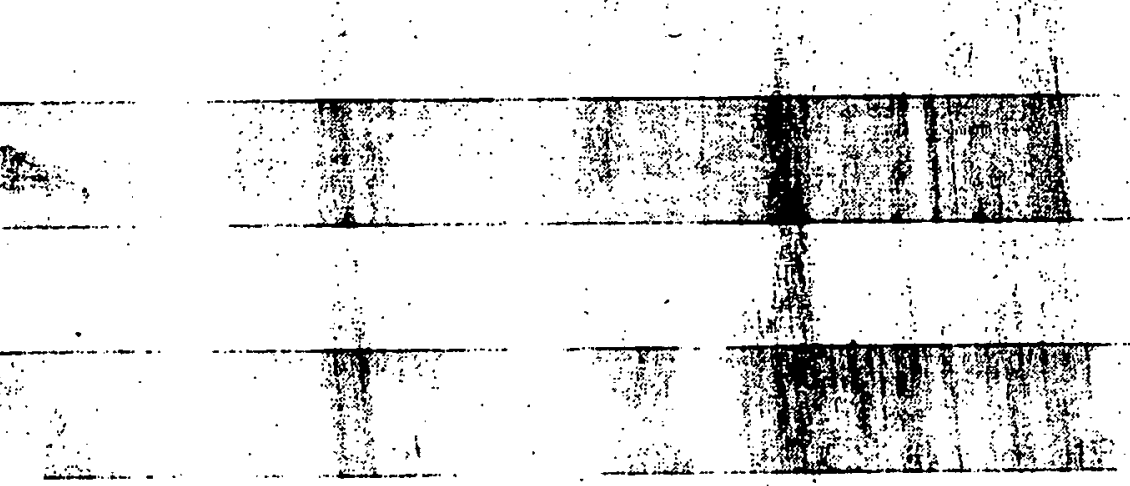

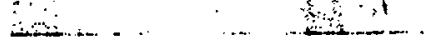

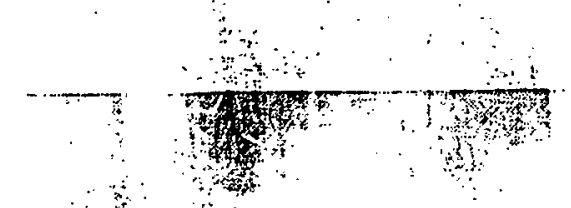




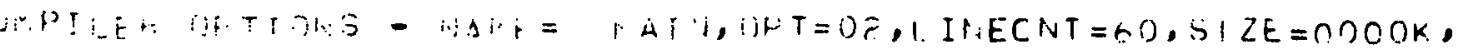

SOLIFCE, EOCOIC, NLLIST, R.OUECK, LOAD, MAP, NOEDIT, NOIL, NOXREF

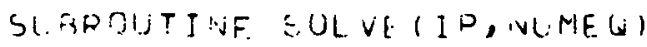

9630

9640

......... steve scilves the conductivity matrix for the nodal temp...

9650

c

9660

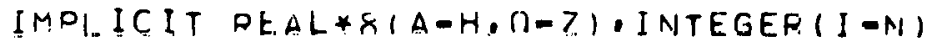

COMMON $\triangle K(240,120), R(1500), T O L, M B A N D, M I T$

COMMON / RLK, NUMBLK, NB, NM, NL, NH, KSHIFT

COMMON/DFICLM/ LELT,TM,JT, JTMAX,IPR

COMMON/TBOUN, CFC(50), TCONV(50), TCONS(1500), NCB, NCBN $(50,4)$,

1 TTYPN(1500)

COMMON/SOLI, HKO(1500),HKB(1500)

THE IJNTFIANGULARIZED STIFFNESS IS STORED ON TAPE 10.

RFWIND 20

$N N=M B A N D$

$N L=N N+1$

$N H=? * M B \triangle N O$

$N B=0$

IF 1 IP $E Q \cdot 2, G O$ TO 90

9680

RFWIND 10

GO TO 25

$10 \mathrm{NE}=\mathrm{NB}+1$

$D G 20 \mathrm{~N}=1, \mathrm{NN}$

$N M=N N+N$

Or, $20 M=2, M E A N O$

$A K(N, M)=A K(N M, M)$

$\approx 0 \quad A K(N M, M)=0.0$

IF (NUMGLK.EQ. NB) GO TO 40

9730

9740

9750

9760

9770

9780

9790

9800

25 CONTINUE

DO $30 \mathrm{~N}=\mathrm{NI}$. NH

30 READ (10) $(A K(N, M), M=1$, MBAND)

IF 1 DELT $E Q \cdot 0 \cdot 0)$ GO TO 32

9900

DO 31 II $=N L, N H$

$I 2=I 1+N N * 1 N B=11$

IF 1 I $\triangle$ GT. NUMEQ , GO TO 31

IF 1 ITYPNAIZI. EQ. 1 I GO TO 31

9820

9830

9840

9850

9860

9870

$A K(I 1,1)=A K(I 1,1)+H K D(I 2) * 1.5$

9880

31 CONTINUE

3?. CONTINUE

IF $(N E \cdot E Q \cdot O$, GO TO 10

9910

9920

9930

9940

9950

9960

9970

9980

9990

$40 D O 70 \mathrm{~N}=1, \mathrm{NN}$

IF $(A K(N, 1), E Q \cdot 0.0$, GO TO 70

10000

DO $60 \mathrm{~L}=2$, MBAND

IF ( DABS (AK $(N, L)) \cdot L E \cdot 1 \cdot D-15)$ GO TO 60

10010

$C=A K(A, L), A K(N, 1)$

10030

$I=N+L=1$

$J=0$

UO $50 k=k$, MBAND

$J=J+1$

$50 \quad A K(I, U)=A K(I, J)-C * A K(N, K)$

$A K(N, E)=C$

10040

10050

10060

10070

10080

10090

10100

DO CONT INUE

70 CONT INUE

10110

DO $80 \mathrm{~N}=1$, NN

XX WFITE (2O) 1 AK (IN,M), $M=1$, MBAND)

IF INIJMIELK. LE. NB I GO TO 170

10130

Si TO 10

10140 


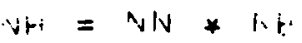

$V N N_{1}=N H-N N / 2$

$N L=N M-1 N N / Z+1$

$101<0$

$1 \cap 170$

KSHTFT $=? * N L-2$ :

10180

10190

Uก $101 I=1, N N \quad$..

10200

li:) READ (20) $(A K(I, J), J=1$, MBANU)

i) $130 I=1, N N$.

IF ( DABS(AK (I, 1)) ILE. 1.D-15) GO TO 130

INDEX $=I+(N B-1)$ *MBANO

IF $($ TNLEX . GE.NUMEQ) GO TO 120

$C P=R($ INDEX)

$R(I N D E X)=C P / A K(I, 1)$

DO $110 \mathrm{~J}=2$, MB-AND

JNDEX $=$ INOEX + J- 1

IF (JNDEX -GT.NUMEQ) GO TO 110

$R(J N D E X)=K(J N D E X) \cdot A K(I ; J) * C P$

110 CONTINUE

$$
\text { GO TO } 130
$$

$1 \angle O$ IF (INDEX - GT.NUMEQ) GO TO 130

$R$, NUMEO $)=R(N U M E Q), A K(I, 1)$

130 CONTINUE

IF (NB.LT. NUMBLK) GO TO 100

DO $131 K 1=Z, N N$

BACKSPACE 20

10220

10240

10250

10260

10270

10280

10290

10300

10310

10320

10330

10340

10350

10360

10370

10380

131 CONTINUE

$14000160 M=1, N N$

$N=N N+1-M$

INDEX = NB MMBAND-M+1

IF (INDEX.GE・NUMEQIGO TO 160

DO $150 K=2$, MBAND

JNDEX $=$ INDEX + K - 1

IF (JNDEX - GT.NUMEQIGO TO 150

$R(I N D E X)=R(I N D E X)-A K(N, K) * R(J N D E X)$

150 CONTINUE

160 CONTINUE

$$
\text { NB } N \text { NE }-1
$$

IF INB .EQ: 0, GO TO $170 \%$

DO $102 \quad K 1=1, N N$

BACKSPACE 20

10390

10400

10410

10420

10430

10440

10450

10460

10470

10480

10490

10500

1 CZ CONTINUE

10510

DO $161 \mathrm{~N}=1, \mathrm{NN}$

161 RFAD (20), $A K(N, M), M=1, M B A N D)$

$D O 163 \quad K 1=1, N N$

BACKSPACE 20

10530

1 IE 3 CONTINUE

GC: TO 140

170 RE TURN

END 